\title{
Poisson brackets, Novikov-Leibniz structures and integrable Riemann hydrodynamic systems
}

Orest D. Artemovych, Denis Blackmore, Anatolij K. Prykarpatski

To cite this article: Orest D. Artemovych, Denis Blackmore, Anatolij K. Prykarpatski (2017) Poisson brackets, Novikov-Leibniz structures and integrable Riemann hydrodynamic systems, Journal of Nonlinear Mathematical Physics 24:1, 41-72, DOI: https://doi.org/10.1080/14029251.2016.1274114

To link to this article: https://doi.org/10.1080/14029251.2016.1274114

Published online: 04 January 2021 


\title{
Poisson brackets, Novikov-Leibniz structures and integrable Riemann hydrodynamic systems
}

\author{
Orest D. Artemovych \\ Institute of Mathematics, Cracow University of Technology, 31-155 Kraków, Poland \\ Denis Blackmore \\ Department of Mathematical Sciences, New Jersey Institute of Technology, \\ Newark, NJ 07102-1982, USA \\ Anatolij K. Prykarpatski \\ Department of Applied Mathematics, AGH University of Science and Technology, \\ 30 Mickiewicz Alley, 30-059 Kraków, Poland
}

Received 4 September 2016

Accepted 22 November 2016

\begin{abstract}
A general differential-algebraic approach is devised for constructing multi-component Hamiltonian operators as differentiations on suitably constructed loop Lie algebras. The related Novikov-Leibniz algebraic structures are presented and a new non-associative "Riemann" algebra is constructed, which is closely related to the infinite multi-component Riemann integrable hierarchies. A close relationship to the standard symplectic analysis techniques is also discussed.

Keywords: Poisson brackets, Hamiltonian operators, differential algebras, differentiations, loop-algebra, 2cocycles, Novikov algebra, right Leibniz algebra, Riemann algebra, Riemann hydrodynamic hierarchy, integrability
\end{abstract}

2000 Mathematics Subject Classification: 35A30, 35G25, 35N10, 37K35, 58J70, 58J72

On memory of a great mathematician and theoretical physicist of the XXth century Nikolai N. Bogolubov (Sr.)

(21 August 1909 - 13 February 1992) who 25 years ago passed away

\section{Introduction}

As is well known $[18,20,36,61]$, many integrable Hamiltonian systems discovered during the last decades were understood owing to the Lie-algebraic properties of their internal hidden symmetry structures. A first account of the Hamiltonian operators and related differential-algebraic structures, lying in the background of integrable systems, was given by I. Gelfand and I. Dorfman $[33,41]$ and later was extended by B. Dubrovin and S. Novikov [34,35], and also by S. Novikov and A. Balinsky [10-13]. Also some new special differential-algebraic techniques [67] were devised for studying the Lax integrability and the structure of related Hamiltonian operators for a wide class of the Riemann type hydrodynamic hierarchies. Just recently considerable work $[6-8,62]$ has been done devoted to the finite dimensional representations of the Novikov algebra. Their importance for constructing integrable multi-component nonlinear Camassa-Holm type dynamical systems on 
functional manifolds was demonstrated by I. Strachan and B. Szablikowski in [78], which in part suggested the Lie-algebraic imbedding of the Novikov algebra into the general Lie-Poisson orbits scheme of classifying Lax integrable Hamiltonian systems. It is also worth of mentioning the related work [44] by Holm and Ivanov in which integrable multicomponent nonlinear Camassa-Holm type dynamical systems on functional manifolds were constructed.

In our work here we describe a differential-algebraic reformulation of the classical Lie algebraic scheme and develop an effective approach to classification of the underlying algebraic structures of integrable multicomponent Hamiltonian systems. In particular, we have devised a simple algorithm allowing to construct new algebraic structures within which the corresponding Hamiltonian operators exist and generate integrable multicomponent dynamical systems. We show, as examples, that the well-known Novikov algebraic structure, obtained in $[12,41]$ as a condition for a matrix differential expression to be Hamiltonian and in $[17,25,47,64]$ as a flat torsion free left-invariant affine connection on affine manifolds, affine structures and convex homogeneous cones, appears in our approach as a differentiation on the Lie-algebra naturally associated with a suitably constructed differential loop algebra. As a direct generalization of this example we obtain two new differentiations, whose underlying algebraic structures coincide, respectively, with the well-known [3,38] right Leibniz algebra, introduced in $[21,22,53]$, and with a new "Riemann" algebra, which naturally generate different Hamiltonian operators describing a wide class of multicomponent hierarchies $[19,67]$ of integrable Riemann hydrodynamic systems. We study the related algebraic structures of the appropriate Leibniz algebra, important for its representations, in some detail. In a supplement, the classical Poisson manifold approach, closely related to our methods for constructing Hamiltonian operators, is also briefly revisited.

\section{The Hamiltonian operators and related algebraic structures via the differential-algebraic approach}

Assume $(\mathbb{A},+, \circ)$ to be a finite-dimensional algebra of dimension $N=\operatorname{dim} \mathbb{A} \in \mathbb{Z}_{+}$(in general noncommutative and non-associative) over an algebraically closed field $\mathbb{K}$. Using the algebra $\mathbb{A}$ one can construct the related loop algebra $\widetilde{\mathbb{A}}$ of smooth mappings $u: \mathbb{S}^{1} \rightarrow \mathbb{A}$ and endow it with a suitably generalized natural convolution $\prec \cdot, \succ$ on $\widetilde{\mathbb{A}}^{*} \times \widetilde{\mathbb{A}} \rightarrow \mathbb{K}$, where $\widetilde{\mathbb{A}}^{*}$ is the corresponding adjoint to $\widetilde{\mathbb{A}}$ space.

First, we shall consider a general scheme of constructing nontrivial ultra-local and local [36] Poisson structures on the adjoint space $\widetilde{\mathbb{A}}^{*}$, compatible with the internal multiplication in the loop algebra $\widetilde{\mathbb{A}}$. Consider a basis $\left\{e_{s} \in \mathbb{A}: s=\overline{1, N}\right\}$ of the algebra $\mathbb{A}$ and its dual $\left\{u^{s} \in \mathbb{A}^{*}: s=\overline{1, N}\right\}$ with respect to $\prec \cdot, \succ$ on $\mathbb{A}^{*} \times \mathbb{A}$, that is $\prec u^{j}, e_{i} \succ:=\delta_{i}^{j}$ for all $i, j=\overline{1, N}$, and such that for any

$$
u(x)=\sum_{s=1, N} u_{s}(x) u^{s} \in \widetilde{\mathbb{A}}^{*}, x \in \mathbb{S}^{1},
$$

the quantities $u_{s}(x):=\prec u(x), e_{s} \succ \in \mathbb{K}$ for all $s=\overline{1, N}, x \in \mathbb{S}^{1}$. Denote by $\widetilde{\mathbb{A}} \wedge \widetilde{\mathbb{A}}:=\operatorname{Skew}(\widetilde{\mathbb{A}} \otimes \widetilde{\mathbb{A}})$ and let $\vartheta^{*}: \widetilde{\mathbb{A}} \wedge \widetilde{\mathbb{A}} \rightarrow \widetilde{\mathbb{A}}$ be a skew-symmetric bilinear mapping. Then the expression

$$
\left\{u_{i}(x), u_{j}(x)\right\}:=\prec u(x), \vartheta^{*}\left(e_{i} \wedge e_{j}\right) \succ
$$

defines for any $x, y \in \mathbb{S}^{1}$ and all $i, j=\overline{1, N}$ an ultra-local linear skew-symmetric pre-Poisson bracket on $\widetilde{\mathbb{A}}^{*}$. Since the algebra $\widetilde{\mathbb{A}}$ possesses its internal multiplicative structure "o", the important problem arises: Under what conditions is the pre-Poisson bracket (2.1) Poisson and compatible with this 
internal structure on $\widetilde{\mathbb{A}}$ ? To proceed with elucidating this question, we define a co-multiplication $\Delta: \widetilde{\mathbb{A}}^{*} \rightarrow \widetilde{\mathbb{A}}^{*} \otimes \widetilde{\mathbb{A}}^{*}$ on any element $u \in \widetilde{\mathbb{A}}^{*}$ by means of the relationship

$$
\prec \Delta(u),(a \otimes b) \succ:=\prec u, a \circ b \succ
$$

for arbitrary $a, b \in \widetilde{\mathbb{A}}$. Note that the co-multiplication $\Delta: \widetilde{\mathbb{A}}^{*} \rightarrow \widetilde{\mathbb{A}}^{*} \otimes \widetilde{\mathbb{A}}^{*}$, defined this way, is a homomorphism of the algebra $\widetilde{\mathbb{A}}^{*}$ with respect to the natural multiplication of functionals, and the linear pre-Poisson structure $\{\cdot, \cdot\} \quad(2.1)$ on $\widetilde{\mathbb{A}}^{*}$ is called compatible with the multiplication " $\circ$ " on the algebra $\widetilde{\mathbb{A}}$, if the following symbolic invariance condition

$$
\Delta\left\{u_{i}(x), u_{j}(x)\right\}=\left\{\Delta\left(u_{i}(x)\right), \Delta\left(u_{j}(x)\right)\right\}
$$

holds for any $x \in \mathbb{S}^{1}$ and all $i, j=\overline{1, N}$.

Taking into account that multiplication in the algebra $\mathbb{A}$ is given for any $i, j=\overline{1, N}$ by the condition

$$
e_{i} \circ e_{j}:=\sum_{s=\overline{1, N}} \sigma_{i j}^{s} e_{s}
$$

where the quantities $\sigma_{i j}^{s} \in \mathbb{K}$ for all $i, j$ and $k=\overline{1, N}$ are constants, the related co-multiplication $\Delta: \widetilde{\mathbb{A}}^{*} \rightarrow \widetilde{\mathbb{A}}^{*} \otimes \widetilde{\mathbb{A}}^{*}$ acts on the basic functionals $u^{s} \in \widetilde{\mathbb{A}}^{*}, s=\overline{1, N}$, as

$$
\Delta\left(u^{s}\right)=\sum_{i, j=1, N} \sigma_{i j}^{s} u^{i} \otimes u^{j} .
$$

Additionally, if the mapping $\vartheta^{*}: \widetilde{\mathbb{A}} \wedge \widetilde{\mathbb{A}} \rightarrow \widetilde{\mathbb{A}}$ is given, for instance, in the simple linear form

$$
\vartheta^{*}:\left(e_{i} \otimes e_{j}-e_{j} \otimes e_{i}\right) \rightarrow \sum_{s=1, N}\left(c_{i j}^{s}-c_{j i}^{s}\right) e_{s},
$$

where quantities $c_{i j}^{s} \in \mathbb{K}$ are constant for all $i, j$ and $s=\overline{1, N}$, then for the adjoint to (2.6) mapping $\vartheta: \operatorname{Symm}\left(\widetilde{\mathbb{A}}^{*}\right) \rightarrow \widetilde{\mathbb{A}}^{*} \wedge \widetilde{\mathbb{A}}^{*}$ one obtains the expression

$$
\vartheta: u^{s} \rightarrow \sum_{i, j=\overline{1, N}}\left(c_{i j}^{s}-c_{j i}^{s}\right) u^{i} \otimes u^{j} .
$$

For the pre-Poisson bracket (2.1) to be a Poisson bracket on $\widetilde{\mathbb{A}}^{*}$, it should must also satisfy the Jacobi identity. To find the corresponding additional constraints on the internal multiplication " $\bigcirc$ " on the algebra $\widetilde{\mathbb{A}}$, define for any $u(x) \in \widetilde{\mathbb{A}}^{*}$ the skew-symmetric linear mapping

$$
\vartheta(u): \widetilde{\mathbb{A}} \rightarrow \widetilde{\mathbb{A}}^{*},
$$

called [41] by the Hamiltonian operator, via the identity

$$
\prec \vartheta(u) a, b \succ:=\prec \vartheta u(x), a \wedge b \succ
$$

for any $a, b \in \widetilde{\mathbb{A}}$, where the mapping $\vartheta: \widetilde{\mathbb{A}}^{*} \rightarrow \widetilde{\mathbb{A}}^{*} \wedge \widetilde{\mathbb{A}}^{*}$ is determined by the expression (2.7) to which it is adjoint. Then it is well known [41] that the pre-Poisson bracket (2.1) is Poisson iff the 
Hamiltonian operator (2.8) satisfies the well known [41] Schouten-Nijenhuis condition:

$$
[[\vartheta(u), \vartheta(u)]]=0
$$

for any $u(x) \in \widetilde{\mathbb{A}}^{*}$. Since the mapping

$$
\vartheta(u) e_{i}=\sum_{s, k=\overline{1, N}}\left(c_{i k}^{s}-c_{k i}^{s}\right) u_{s}(x) u^{k}
$$

holds for any basis element $e_{i} \in \mathbb{A}, i=\overline{1, N}$, the resulting linear pre-Poisson bracket (2.1) becomes equal to

$$
\begin{aligned}
\left\{u_{i}(x), u_{j}(x)\right\} & =\prec \vartheta(u) e_{i}, e_{j} \succ= \\
=\sum_{s=1, N}\left(c_{i j}^{s}-c_{j i}^{s}\right) u_{s}(x) & =\prec u(x), \sum_{s=1, N}\left(c_{i j}^{s}-c_{j i}^{s}\right) e_{s}>
\end{aligned}
$$

for any $u(x) \in \widetilde{\mathbb{A}}^{*}$. Now, defining on the algebra $\mathbb{A}$ the naturally adjacent to the algebra $\mathbb{A}$ Lie commutator structure

$$
\left[e_{i}, e_{j}\right]=e_{i} \circ e_{j}-e_{j} \circ e_{i}:=\sum_{s=\overline{1, N}}\left(c_{i j}^{s}-c_{j i}^{s}\right) e_{s}
$$

for any basis elements $e_{i}, e_{j} \in \mathbb{A}, i, j=\overline{1, N}$, the expression (2.12) yields the well known [1,4] classical Lie-Poisson bracket

$$
\left\{u_{i}(x), u_{j}(x)\right\}=\prec u,\left[e_{i}, e_{j}\right] \succ
$$

Concerning the adjacent Lie algebra structure condition (2.13), it can be easily rewritten as the set of relationships,

$$
\sigma_{i j}^{s}-\sigma_{j i}^{s}=c_{i j}^{s}-c_{j i}^{s}
$$

whose evident solution is

$$
c_{i j}^{s}=\sigma_{i j}^{s}
$$

for any $i, j, s=\overline{1, N}$. It is also clear that the compatibility condition (2.3) is completely unnecessary $[11,13]$ for the pre-Poisson bracket $(2.1)$ to be a Poisson one. Moreover, as the bracket $(2.14)$ is of the classical Lie-Poisson type, for the Hamiltonian operator (2.11) to satisfy the Schouten-Nijenhuis condition (2.10) is enough to check only the weak Jacobi identity for the weak Lie algebra $\mathscr{L}_{\widetilde{\mathbb{A}}}$, adjacent to the algebra $\widetilde{\mathbb{A}}$ via imposing the Lie structure (2.13), taking into account the relationships 
(2.16). Simple calculations for the special skew-symmetric case

$$
e_{i} \circ e_{j}+e_{j} \circ e_{i}=0
$$

for all $i, j=\overline{1, N}$ give rise to the constraints

$$
e_{i} \circ e_{j}+e_{j} \circ e_{i}=0,\left(e_{i} \circ e_{j}\right) \circ e_{k}+\left(e_{j} \circ e_{k}\right) \circ e_{i}+\left(e_{k} \circ e_{i}\right) \circ e_{j}=0,
$$

coinciding exactly with those in [41]. The corresponding Hamiltonian operator (2.8) then acts as

$$
\vartheta(u) e_{i}=\sum_{s, k=1, N}\left(\sigma_{i k}^{s}-\sigma_{k i}^{s}\right) u_{s}(x) u^{k}
$$

on any basis element $e_{i} \in \mathbb{A}$. Since the bracket (2.14), owing to the constraints (2.17) and (2.18), satisfies the weak Jacobi identity implying that the mapping $\vartheta(u): \widetilde{\mathbb{A}} \rightarrow \widetilde{\mathbb{A}}^{*}$ satisfies the SchoutenNijenhuis condition (2.10), one has the following result.

Theorem 2.1. The general linear pre-Poisson bracket (2.1) on $\widetilde{\mathbb{A}}^{*}$ under the constraints (2.17) and (2.18) on the algebra $\mathbb{A}$, which is of the Lie-Poisson type on the adjoint space $\mathscr{L}_{\widetilde{\mathbb{A}}}^{*}$ to the weak Lie algebra $\mathscr{L}_{\widetilde{\mathbb{A}}}$ adjacent to the loop algebra $\widetilde{\mathbb{A}}$, is a priori a Poisson and compatible with the internal algebraic structure of $\mathbb{A}$.

Remark 2.1. Similarly, one can consider a simple ultra-local quadratic pre-Poisson bracket on $\widetilde{\mathbb{A}}^{*}$,

$$
\left\{u_{i}(x), u_{j}(x)\right\}:=\prec u(x) \otimes u(x), \vartheta^{*}\left(e_{i} \wedge e_{j}\right) \succ,
$$

where the skew-symmetric mapping $\vartheta^{*}: \widetilde{\mathbb{A}} \wedge \widetilde{\mathbb{A}} \rightarrow \operatorname{Symm}(\widetilde{\mathbb{A}} \otimes \widetilde{\mathbb{A}})$ is given for any $i, j=\overline{1, N}$ in the quadratic form

$$
\vartheta^{*}\left(e_{i} \otimes e_{j}-e_{j} \otimes e_{i}\right):=\sum_{k, s=\overline{1, N}}\left(c_{i j}^{k s}-c_{j i}^{k s}\right)\left(e_{k} \otimes e_{s}+e_{s} \otimes e_{k}\right) .
$$

In particular, if to assume that the coefficients $c_{i j}^{k s}=\sigma_{i j}^{k} \alpha^{s}$ for some constant numbers $\sigma_{i j}^{k}$ and $\alpha^{s} \in \mathbb{K}$ for all $i, j$ and $k, s=\overline{1, N}$, where

$$
e_{k} \circ e_{s}:=\sum_{k=1, N} \sigma_{i j}^{k} e_{k}
$$

then the pre-Poissson bracket (2.20) yields a very compact form

$$
\left\{u_{i}(x), u_{j}(x)\right\}:=\prec u(x) \otimes u(x), \alpha \otimes\left[e_{i}, e_{j}\right]+\left[e_{i}, e_{j}\right] \otimes \alpha \succ,
$$

generalizing (2.14) and parametrically depending on the constant vector

$$
\alpha:=\sum_{s=\overline{1, N}} \alpha^{s} e_{s} \in \mathbb{A} .
$$

For the pre-Poisson bracket (2.22) to be Poisson one can formulate suitable constraints on the algebraic structure of $\widetilde{\mathbb{A}}$, similar to those obtained in [11], which we shall not consider here.

Now, let $\widetilde{\mathbb{A}}(u) \subset \widetilde{\mathbb{A}}$ denote the polynomial differential ideal generated by an element $u \in \widetilde{\mathbb{A}}$ and its derivatives $D_{x}^{n} u \in \widetilde{\mathbb{A}}, n \in \mathbb{Z}_{+}$. The corresponding space of polynomial functions $\widetilde{\mathbb{A}}(u) \rightarrow \mathbb{K}$, constructed by means of some scalar form on $\widetilde{\mathbb{A}}(u)$, will be denoted by $\mathscr{F}_{\widetilde{\mathbb{A}}}(u)$. Then the basic 
ultra-local and linear, with respect to an independent element $u(x) \in \widetilde{\mathbb{A}}, x \in \mathbb{S}^{1}$, pre-Poisson bracket (2.1) is easily generalized to a local pre-Poisson bracket for arbitrary functions $f, g \in \mathscr{F}_{\widetilde{\mathbb{A}}}(u)$ :

$$
\{f, g\}(u)=\prec u(x), \vartheta^{*}(\nabla f(u(x)) \wedge \nabla g(u(x) \succ,
$$

in which the mapping $\vartheta^{*}: \widetilde{\mathbb{A}} \wedge \widetilde{\mathbb{A}} \rightarrow \operatorname{Symm}(\widetilde{\mathbb{A}} \otimes \widetilde{\mathbb{A}})$ is invariantly reduced on the subspace $\widetilde{\mathbb{A}}(u) \wedge$ $\widetilde{\mathbb{A}}(u)$ and depends nontrivially on the differentiation $D_{x}: \widetilde{\mathbb{A}} \rightarrow \widetilde{\mathbb{A}}$. In (2.23), we denoted the usual linear gradient mapping from $\mathscr{F}_{\widetilde{\mathbb{A}}}(u)$ to the ideal $\widetilde{\mathbb{A}}(u) \subset \widetilde{\mathbb{A}}$ by " $\nabla$ ", that is for a given $h \in \mathscr{F}_{\widetilde{\mathbb{A}}}(u)$, $\nabla h(u(x)) \in \widetilde{\mathbb{A}}(u)$ and

$$
\prec v(x), \nabla h\left(u(x) \succ:=d h(u+\varepsilon v) /\left.d \varepsilon\right|_{\varepsilon=0}\right.
$$

for any $v(x) \in \widetilde{\mathbb{A}}^{*}, x \in \mathbb{S}^{1}$. Keeping in mind the problem of finding constraints on the multiplicative structure of the algebra $\widetilde{\mathbb{A}}$ under which the pre-Poisson bracket (2.23) is Poisson, it is very interesting to construct nontrivial examples of linear local pre-Poisson brackets on $\mathscr{F}_{\widetilde{\mathbb{A}}}(u)$, compatible with the multiplication " $\circ$ " on $\mathbb{A}$ and nontrivially depending on the usual differential operator $D_{x}: \widetilde{\mathbb{A}} \rightarrow \widetilde{\mathbb{A}}$ for $x \in \mathbb{S}^{1}$. In particular, for arbitrary functions $f, g \in \mathscr{F}_{\widetilde{\mathbb{A}}}(u)$ one can consider the following nontrivial and simplest linear local pre-Poisson bracket

$$
\{f, g\}(u):=\prec u(x), \vartheta^{*}(\nabla f(u(x)) \wedge \nabla g(u(x)) \succ,
$$

where

$$
\vartheta^{*}:(a(x) \wedge b(x)) \rightarrow \sum_{j, k, s=\overline{1, N}}\left[c_{j k}^{s} D_{x} a^{j}(x) b^{k}(x)-c_{j k}^{s} D_{x} b^{j}(x) a^{k}(x)\right] e_{s}
$$

for any

$$
a(x):=\sum_{j=\overline{1, N}} a^{j}(x) e_{j}, b(x):=\sum_{j=\overline{1, N}} b^{j}(x) e_{j} \in \widetilde{\mathbb{A}}, x \in \mathbb{S}^{1}
$$

and some arbitrarily chosen constant quantities $c_{j k}^{s} \in \mathbb{K}$ for all $j, k$ and $s=\overline{1, N}$. If one also assumes that these constant quantities satisfy the condition (2.16), that is $c_{i j}^{s}=\sigma_{i j}^{s}$ for all $i, j$ and $s=\overline{1, N}$, the mapping (2.25) can be recast as

$$
\vartheta^{*}:(a(x) \wedge b(x)) \rightarrow D_{x} a(x) \circ b(x)-D_{x} b(x) \circ a(x),
$$

providing the pre-Poisson bracket (2.24) for arbitrary functions $f, g \in \mathscr{F}_{\widetilde{\mathbb{A}}}(u)$ with the canonical Lie-Poisson form

$$
\{f, g\}(u):=\prec u(x), D_{x} \nabla f(u(x)) \circ \nabla g(u(x))-D_{x} \nabla g(u(x)) \circ \nabla f(u(x) \succ,
$$

which was recently presented in [78]. Thus, if the Lie structure

$$
[a(x), b(x)]_{D}:=D_{x} a(x) \circ b(x)-D_{x} b(x) \circ a(x)
$$

for any $a(x), b(x) \in \widetilde{\mathbb{A}}, x \in \mathbb{S}^{1}$, generates the weak adjacent Lie algebra $\mathscr{L}_{\widetilde{\mathbb{A}}}$, the pre-Poisson bracket (2.27) will automatically be Poisson on the space $\mathscr{F}_{\widetilde{\mathbb{A}}}(u)$. Moreover, the expression (2.27), rewritten 
in the tensor form

$$
\begin{aligned}
\{f, g\}(u) & =\prec \Delta u(x), D_{x} \nabla f(u(x)) \otimes \nabla g(u(x))- \\
-D_{x} \nabla g(u(x)) \otimes \nabla f(u(x) \succ & :=(\vartheta(u) \nabla f(u(x)), \nabla g(u(x))),
\end{aligned}
$$

naturally defines a related bilinear form $(\cdot, \cdot)$ on the weak adjacent Lie algebra $\mathscr{L}_{\widetilde{A}}$, allowing to determine the corresponding Hamiltonian operator $\vartheta(u): \mathscr{L}_{\widetilde{\mathbb{A}}} \rightarrow \mathscr{L}_{\widetilde{\mathbb{A}}}$, whose matrix representation with respect to the basis $\left\{e_{s} \in \mathbb{A}: s=\overline{1, N}\right\}$ is

$$
\vartheta(u)=\sigma(u) D_{x}+D_{x} \sigma(u)^{\top}
$$

where

$$
\sigma(u):=\left\{\sum_{s=\overline{1, N}} \sigma_{i j}^{s} u_{s}: i, j=\overline{1, N}\right\} .
$$

So, if the Hamiltonian operator (2.30) satisfies the Schouten-Nijenhuis condition (2.10), the prePoisson bracket (2.29) is Poisson. Yet, simultaneously, if the adjacent Lie algebra structure (2.28) satisfies the weak Jacobi condition

$$
\begin{aligned}
\prec u(x),\left[[a(x), b(x)]_{D}, c(x)\right]_{D} & \succ \prec u(x),\left[[b(x), c(x)]_{D}, a(x)\right]_{D} \succ \\
+\prec u(x),\left[[c(x), a(x)]_{D}, b(x)\right]_{D} & \succ=0
\end{aligned}
$$

for any elements $a(x), b(x)$ and $c(x) \in \widetilde{\mathbb{A}}, x \in \mathbb{S}^{1}$, then the pre-Poisson bracket (2.27) equivalent to (2.29), being of the Lie-Poisson type, will be a priori Poisson. As the second criterion is easier to check, after some simple calculations one obtains the well-known [12,41] Novikov algebra constraints

$$
\left[R_{a}, R_{b}\right]=0,\left[L_{a}, L_{b}\right]=L_{[a, b]}
$$

on the multiplication structure of the algebra $\mathbb{A}$, where, by definition, for any $a, b \in \mathbb{A}$ the bracket $[a, b]:=a \circ b-b \circ a$ and the mappings $L_{a}, R_{a}: \mathbb{A} \rightarrow \mathbb{A}$ are left and right multiplications, respectively: $L_{a} b:=a \circ b=R_{b} a$.

Remark 2.2. As follows from Proposition 3.1, formulated below, if the algebra $\mathbb{A}$ is a Novikov algebra (2.32), then the constructed above Hamiltonian operator $\vartheta(u): \mathscr{L}_{\widetilde{\mathbb{A}}} \rightarrow \mathscr{L}_{\widetilde{\mathbb{A}}}$ is a differentiation of the weak adjacent Lie algebra $\mathscr{L}_{\widetilde{\mathbb{A}}}$ and vice versa: the operator $\vartheta(u): \mathscr{L}_{\widetilde{\mathbb{A}}} \rightarrow \mathscr{L}_{\widetilde{\mathbb{A}}}$ is Hamiltonian if it is a differentiation of the weak Lie algebra $\mathscr{L}_{\widetilde{\mathbb{A}}}$ adjacent to the Novikov algebra (2.32).

The next example of the bilinear, local and weakly skew-symmetric mapping

$$
\vartheta^{*}:(a(x) \wedge b(x)) \rightarrow D_{x}^{-1} a(x) \circ b(x)-D_{x}^{-1} b(x) \circ a(x),
$$

where $D_{x} D_{x}^{-1}:=1: \widetilde{\mathbb{A}} \rightarrow \widetilde{\mathbb{A}}$ is the identity mapping, generates the weak adjacent Lie algebra $\mathscr{L}_{\widetilde{\mathbb{A}}}$ structure

$$
[a(x), b(x)]_{D}:=D_{x}^{-1} a(x) \circ b(x)-D_{x}^{-1} b(x) \circ a(x)
$$


for any $a(x), b(x) \in \widetilde{\mathbb{A}}$. It is easy to check that the commutator structure (2.34) satisfies the weak Jacobi identity (2.31) iff the multiplicative structure of the algebra $\mathbb{A}$ satisfies the so called [53] right Leibniz algebra constraints:

$$
R_{a \circ b}=\left[R_{a}, R_{b}\right], \quad R_{a \circ b}+R_{b \circ a}=0
$$

for arbitrary elements $a, b \in \mathbb{A}$. The corresponding matrix integro-differential Hamiltonian operator on the space $\mathscr{F}_{\widetilde{\mathbb{A}}}(u)$ with respect to the basis $\left\{e_{s} \in \mathbb{A}: s=\overline{1, N}\right\}$ for this case equals

$$
\vartheta(u)=\sigma(u) D_{x}^{-1}+D_{x}^{-1} \sigma(u)^{\top}
$$

for any $u(x) \in \widetilde{\mathbb{A}}^{*}, x \in \mathbb{S}^{1}$.

Consider now the bilinear, local and weakly skew-symmetric mapping

$$
\left.\left.\vartheta^{*}:(a(x) \wedge b(x)) \rightarrow D_{x}^{-1} b(x) \circ D_{x} a(x)\right)-D_{x}^{-1} a(x) \circ D_{x} b(x)\right),
$$

which naturally generates the adjacent Lie algebra $\mathscr{L}_{\widetilde{\mathbb{A}}}$ structure

$$
[a(x), b(x)]_{D}:=D_{x}^{-1} b(x) \circ D_{x} a(x)-D_{x}^{-1} a(x) \circ D_{x} b(x) .
$$

Then it is easy to check that the commutator structure (2.38) satisfies the weak Jacobi identity (2.31), iff the following so called Riemann algebra $\mathbb{A}$ multiplicative structure

$$
\left[R_{a}, R_{b}\right]=0, L_{a \circ b}=R_{a \circ b}=L_{b \circ a}
$$

holds for arbitrary elements $a, b \in \mathbb{A}$. For the related Hamiltonian operator on the functional space $\mathscr{F}_{\widetilde{\mathbb{A}}}(u)$ with respect to the basis $\left\{e_{s} \in \mathbb{A}: s=\overline{1, N}\right\}$ one easily obtains from (2.37) the integrodifferential expression

$$
\vartheta(u)=D_{x} \sigma(u) D_{x}^{-1}-D_{x}^{-1} \sigma(u)^{\top} D_{x}
$$

for any $u(x) \in \widetilde{\mathbb{A}}^{*}, x \in \mathbb{S}^{1}$. The above results can be reformulated as the following theorem.

Theorem 2.2. An arbitrary linear pre-Poisson bracket (2.29) on the functional space $\mathscr{F}_{\widetilde{\mathbb{A}}}(u)$, which is of the Lie-Poisson type on the adjoint space $\mathscr{L}_{\widetilde{\mathbb{A}}}^{*}$ to the weak Lie algebra $\mathscr{L}_{\widetilde{\mathbb{A}}}$ adjacent to the loop algebra $\widetilde{\mathbb{A}}$, is a priori Poisson and compatible with the internal structure of the algebra $\mathbb{A}$ iff the related Lie algebra structure $[\cdot, \cdot]_{D}$ satisfies the weak Jacobi condition.

Thus, all the operators (2.30), (2.36) and (2.40) are Hamiltonian and a priori satisfy the Schouten-Nijenhuis condition (2.10), as easily follows from Theorem 2.2. It is also clear that in contrast to the simple Hamiltonian operator criterion formulated in this theorem, direct and very cumbersome checking of the Schouten-Nijenhuis condition as in [41] for the cases considered above, would be required for the multiplicative structures on the algebra $\mathbb{A}$ coinciding with (2.32), (2.35) and (2.39).

\section{Hamiltonian operators and related algebraic structures via the Lie-algebraic approach}

Assume now that the loop algebra $(\widetilde{\mathbb{A}},+, \circ)$ allows an weak adjacent Lie algebra extension $\left(\mathscr{L}_{\widetilde{\mathbb{A}}},+,[\cdot, \cdot]_{D}\right)$ by means of a commutator operation $[\cdot, \cdot]_{D}: \widetilde{\mathbb{A}} \times \widetilde{\mathbb{A}} \rightarrow \widetilde{\mathbb{A}}$, which can be endowed 
$[15,16,36,76]$ with a nondegenerate ad-invariant

$$
\left([a, b]_{D}, c\right)=\left(a,[b, c]_{D}\right)
$$

and symmetric

$$
(a, b)=(b, a)
$$

bilinear form $(\cdot, \cdot): \mathscr{L}_{\widetilde{\mathbb{A}}} \times \mathscr{L}_{\widetilde{\mathbb{A}}} \rightarrow \mathbb{K}$ for any $a, b$ and $c \in \mathscr{L}_{\mathbb{A}}$.

Remark 3.1. If the Lie-structure $[\cdot, \cdot]_{D}$ on $\widetilde{\mathbb{A}}$ coincides with the usual commutator structure $[\cdot, \cdot]$ on $\widetilde{\mathbb{A}}$, with respect to the multiplication " $\circ$ ", and the symmetric bilinear form (3.2) also satisfies the shifting property

$$
(a \circ b, c)=(a, b \circ c)
$$

for any $a, b$ and $c \in \mathscr{L}_{\widetilde{\mathbb{A}}}$, then the ad-invariance condition (3.1) automatically holds.

The form (3.2) makes it possible to construct the natural identification $\mathscr{L}_{\widetilde{\mathbb{A}}}^{*} \simeq \mathscr{L}_{\widetilde{\mathbb{A}}}$. One can consider the generated by an element $u \in \mathscr{L}_{\widetilde{\mathbb{A}}}^{*}$ subspace of polynomial functions $\mathscr{F}_{\widetilde{\mathbb{A}}}(u) \subset \mathscr{F}_{\widetilde{\mathbb{A}}}\left(\mathscr{L}_{\widetilde{\mathbb{A}}}^{*}\right)$ of the space $\mathscr{F}\left(\mathscr{L}_{\mathbb{A}}^{*}\right)$ of smooth functions on $\mathscr{L}_{\mathbb{A}}^{*}$, jointly with its related Lie-Poisson bracket:

$$
\{f, g\}_{0}:=\left(u,[\nabla f(u), \nabla g(u)]_{D}\right)
$$

for any $f, g \in \mathscr{F}_{\widetilde{\mathbb{A}}}(u)$. Owing to the construction $[1,4,15,18,36]$, the Lie-Poisson bracket (3.4) satisfies a priori the classical Jacobi identity, and it can serve as a very powerful tool for constructing the related Hamiltonian operators on the functional space $\mathscr{F}_{\widetilde{\mathbb{A}}}(u)$. In particular, following $[41,60]$, a smooth mapping $\vartheta(u): \mathscr{L}_{\widetilde{\mathbb{A}}} \rightarrow \mathscr{L}_{\widetilde{\mathbb{A}}}^{*} \simeq \mathscr{L}_{\widetilde{\mathbb{A}}}$ for a chosen element $u \in \widetilde{\mathbb{A}}$ is a Hamiltonian operator if the related pre-Poisson bracket

$$
\{f, g\}:=(\vartheta(u) \nabla f(u), \nabla g(u)),
$$

determined for any $f, g \in \mathscr{F}_{\widetilde{\mathbb{A}}}(u)$, satisfies the Jacobi identity.

Taking into account that the canonical Lie-Poisson bracket (3.4) can be, in general, local involving essentially the loop Lie algebra structure of $\mathscr{L}_{\widetilde{\mathbb{A}}}$, we proceed further to extending the Lie algebra structure on $\mathscr{L}_{\widetilde{\mathbb{A}}}$ by means of the standard [36] central extension technique. Namely, let $\hat{\mathscr{L}}_{\widetilde{\mathbb{A}}}:=\mathscr{L}_{\widetilde{\mathbb{A}}} \oplus \mathbb{K}$ denote the centrally extended Lie algebra $\mathscr{L}_{\widetilde{\mathbb{A}}}$ endowed with the bracket

$$
[(a ; \alpha),(b ; \beta)]_{D}:=\left([a, b]_{D} ; \varpi_{2}(a, b)\right)
$$

for any $a, b \in \mathscr{L}_{\widetilde{\mathbb{A}}}$ and $\alpha, \beta \in \mathbb{K}$, where the 2-cocycle $\varpi_{2}: \mathscr{L}_{\widetilde{\mathbb{A}}} \times \mathscr{L}_{\widetilde{\mathbb{A}}} \rightarrow \mathbb{K}$ is a skew-symmetric bilinear form satisfying the Jacobi identity:

$$
\varpi_{2}\left([a, b]_{D}, c\right)+\varpi_{2}\left([b, c]_{D}, a\right)+\varpi_{2}\left([c, a]_{D}, b\right)=0
$$

for any $a, b$ and $c \in \mathscr{L}_{\widetilde{\mathbb{A}}}$. It is evident that the existence of nontrivial central extensions on the Lie algebra $\mathscr{L}_{\widetilde{\mathbb{A}}}$ strongly depends on the algebraic structure of the algebra $\mathbb{A}$ underlying the whole construction presented above. Yet there exist some general algebraic properties which allow to proceed 
further with success. For example, assume that a smooth mapping D : $\mathscr{L}_{\widetilde{\mathbb{A}}} \rightarrow$ End $\mathscr{L}_{\widetilde{\mathbb{A}}}$ defines for any $u \in \mathscr{L}_{\widetilde{\mathbb{A}}}^{*} \simeq \mathscr{L}_{\widetilde{\mathbb{A}}}$ a weak differentiation of the Lie algebra $\mathscr{L}_{\widetilde{\mathbb{A}}}$, that is

$$
\left(c, \mathrm{D}_{u}[a, b]_{D}\right)=\left(c,\left[\mathrm{D}_{u} a, b\right]_{D}+\left[a, \mathrm{D}_{u} b\right]_{D}\right)
$$

for any $a, b$ and $c \in \mathscr{L}_{\widetilde{\mathbb{A}}}$. Then the following important proposition $[59,76]$ holds.

Proposition 3.1. Let a linear mapping $\mathrm{D}_{u}: \mathscr{L}_{\widetilde{\mathbb{A}}} \rightarrow \mathscr{L}_{\widetilde{\mathbb{A}}}$ be for a fixed $u \in \mathscr{L}_{\widetilde{\mathbb{A}}}^{*}$ a weak skewsymmetric differentiation of the Lie algebra $\mathscr{L}_{\widetilde{\mathbb{A}}}$. Then the expression

$$
\varpi_{2}(a, b):=\left(a, \mathrm{D}_{u} b\right)
$$

for any $a, b \in \mathscr{L}_{\widetilde{\mathbb{A}}}$ and $u \in \mathscr{L}_{\widetilde{\mathbb{A}}}^{*} \simeq \mathscr{L}_{\widetilde{\mathbb{A}}}$ defines a nontrivial 2-cocycle on the Lie algebra $\mathscr{L}_{\widetilde{\mathbb{A}}}$.

A proof is given by means of direct checking the Jacobi identity (3.7) and is omitted.

Remark 3.2. The expression (3.9) when linear in the element $u \in \mathscr{L}_{\widetilde{\mathbb{A}}}^{*}$ can be, evidently, represented for any $a, b \in \widetilde{\mathbb{A}}$ in the following scalar form:

$$
\left(a, \mathrm{D}_{u} b\right)=\left(u, \vartheta^{*}(a \wedge b)\right),
$$

where $\vartheta^{*}: \widetilde{\mathbb{A}} \wedge \widetilde{\mathbb{A}} \rightarrow \widetilde{\mathbb{A}}$ is some bilinear skew-symmetric mapping.

As follows from the results of Section (2) the right-hand side of expression (3.10) allows the equivalent form

$$
\left(u, \vartheta^{*}(a \wedge b)\right)=\left(u,[a, b]_{\bar{D}}\right),
$$

where the bracket $[a, b]_{\bar{D}}$ defines for any $a, b \in \widetilde{\mathbb{A}}$ a new adjacent Lie algebra structure on the loop algebra $\widetilde{\mathbb{A}}$, a priori compatible with the basic Lie structure $[\cdot, \cdot]_{D}$. In the case when these Lie structures coincide, that is $[\cdot, \cdot]_{D}=[\cdot, \cdot]_{\bar{D}}$, the cocycle (3.9) naturally determines on the phase space $\mathscr{F}_{\widetilde{A}}(u)$ the equivalent to (3.4) Poisson bracket

$$
\{f, g\}(u):=\left(\nabla f(u(x)), \mathrm{D}_{u} \nabla g(u(x))\right)
$$

for any $f, g \in \mathscr{F}_{\widetilde{\AA}}(u)$. Moreover, as follows from (3.11) and the ad-invariance property (3.1), the mapping $\mathrm{D}_{u}(\cdot)=-[u, \cdot]_{D}$ for any $u \in \mathscr{L}_{\widetilde{\mathbb{A}}}^{*}$ is automatically a derivation of the weak adjacent Lie algebra $\mathscr{L}_{\widetilde{\mathbb{A}}}$.

Example 3.1. As a natural example of the derivation $\mathrm{D}_{u}: \mathscr{L}_{\widetilde{\mathbb{A}}} \rightarrow \mathscr{L}_{\widetilde{\mathbb{A}}}$ one can take the mapping

$$
\mathrm{D}_{u}:=\Delta_{1} u(x) D_{x}+D_{x} \Delta_{2} u(x)
$$

where for $u \in \mathscr{L}_{\widetilde{\mathbb{A}}}^{*}$ the expressions $\Delta_{j} u(x): \widetilde{\mathbb{A}} \rightarrow \widetilde{\mathbb{A}}^{*}, j=\overline{1,2}$, denote the convolution operators of the co-multiplication $\Delta: \widetilde{\mathbb{A}}^{*} \rightarrow \widetilde{\mathbb{A}}^{*} \otimes \widetilde{\mathbb{A}}^{*}$ with respect to its first and second tensor components, 
respectively. In particular, we have

$$
(\Delta u, a \otimes b):=(u, a \circ b)=\sum_{s, i, j=1}^{N} \sigma_{i j}^{s} \int_{\mathbb{S}^{1}} u_{s}(x) a^{i}(x) b^{j}(x) d x
$$

for a fixed $u=\sum_{s=1}^{N} u_{s}(x) u^{j} \in \widetilde{\mathbb{A}}^{*} \simeq \widetilde{\mathbb{A}}$ and any

$$
a=\sum_{i=1}^{N} a^{i}(x) e_{i}, b=\sum_{j=1}^{N} b^{j}(x) e_{j} \in \mathscr{L}_{\widetilde{\mathbb{A}}}
$$

as we have assumed by definition, that $\left(u^{i}, e_{j}\right):=\delta_{j}^{i}, i, j=\overline{1, N}$. If now the weak Lie algebra $\mathscr{L}_{\widetilde{\mathbb{A}}}$ is generated by the commutator Lie structure (3.4), that is

$$
[a, b]_{D}=D_{x} a \circ b-D_{x} b \circ a
$$

for any $a, b \in \mathscr{L}_{\widetilde{\mathbb{A}}}$, it easy to check that the mapping (3.13) is a skew-symmetric with respect to the bilinear form $(\cdot, \cdot)$ on $\widetilde{\mathbb{A}}$ weak differentiation of the Lie algebra $\mathscr{L}_{\widetilde{\mathbb{A}}}$. Moreover, the related weak Lie algebraic structure $[\cdot, \cdot]_{D^{\prime}}$ on $\widetilde{\mathbb{A}}$, satisfying the condition $\left(u,[a, b]_{D^{\prime}}\right)=\left(a, \mathrm{D}_{u} b\right)$ for any $a, b \in \mathscr{L}_{\widetilde{\mathbb{A}}}$, coincides exactly with that (3.14).

There are also other strictly algebraic tools for constructing Poisson brackets on the functional space $\mathscr{F}_{\widetilde{\mathbb{A}}}(u)$. For instance, as a simple consequence of Proposition 3.1 the following result $[33,59$, 76] holds.

Proposition 3.2. Let a nondegenerate linear skew-symmetric $\mathscr{R}$-matrix endomorphism $\mathscr{R}: \mathscr{L}_{\widetilde{\mathbb{A}}} \rightarrow$ $\mathscr{L}_{\widetilde{\mathbb{A}}}$ satisfy the well-known weak Yang-Baxter commutator condition:

$$
\left(c,[\mathscr{R} a, \mathscr{R} b]_{D}\right)=\left(c, \mathscr{R}[\mathscr{R} a, b]_{D}+[a, \mathscr{R} b]_{D}\right)
$$

for any $a, b$ and $c \in \mathscr{L}_{\widetilde{\mathbb{A}}}$. Then the inverse mapping $\mathscr{R}^{-1}: \mathscr{L}_{\widetilde{\mathbb{A}}} \rightarrow \mathscr{L}_{\widetilde{\mathbb{A}}}$ is a weak skew-symmetric differentiation of the Lie algebra $\mathscr{L}_{\widetilde{\mathbb{A}}}$ and the expression

$$
\varpi_{2}(a, b)=\left(a, \mathscr{R}^{-1} b\right)
$$

defines for any $a, b \in \mathscr{L}_{\widetilde{\mathbb{A}}}$ a 2-cocycle on $\mathscr{L}_{\widetilde{\mathbb{A}}}$.

Consider for any function $f \in \mathscr{F}_{\widetilde{\mathbb{A}}}(u)$ its differential $\delta f \in \Lambda^{1}(\widetilde{\mathbb{A}}(u))$ and define for a chosen element $h \in \widetilde{\mathbb{A}}(u)^{*}$ the vector field $\xi_{h} \in \Gamma(\widetilde{\mathbb{A}}(u))$ via the equality

$$
\delta f\left(\xi_{h}\right)=(\nabla f(u), h)
$$

As symplectic forms on the phase space $\widetilde{\mathbb{A}}(u)$ are dual objects to the Poisson brackets on the functional space $\mathscr{F}_{\widetilde{\mathbb{A}}}(u)$, one easily obtains the following $[59,75,76]$ proposition. 
Proposition 3.3. Assume that the Lie algebra $\mathscr{L}_{\widetilde{\mathbb{A}}}$ allows a skew-symmetric nondegenerate $\mathscr{R}$ structure homomorphism $\mathscr{R}: \mathscr{L}_{\widetilde{\mathbb{A}}} \rightarrow \mathscr{L}_{\widetilde{\mathbb{A}}}$, satisfying the generalized weak Yang-Baxter condition

$$
\left(c, \mathscr{R}\left([\mathscr{R} a, b]_{D}+[a, \mathscr{R} b]_{D}\right)-[\mathscr{R} a, \mathscr{R} b]_{D}\right)=\alpha\left(c,[a, b]_{D}\right)
$$

for any $a, b$ and $c \in \mathscr{L}_{\widetilde{\mathbb{A}}}$ and some $\alpha \in \mathbb{K} \backslash\{0\}$. Then differential 2-forms $\omega_{j}^{(2)} \in \Lambda^{2}(\widetilde{\mathbb{A}}(u)), j=\overline{1,2}$, defined on the ideal $\widetilde{\mathbb{A}}(u) \subset \widetilde{\mathbb{A}}^{*}$ as

$$
\omega_{1}^{(2)}\left(\xi_{f}, \xi_{g}\right):=\left(\nabla f(u), \mathscr{R}^{-1} \nabla g(u)\right)
$$

and

$$
\omega_{2}^{(2)}\left(\xi_{f}, \xi_{g}\right):=\left(u,[\mathscr{R} \nabla f(u), \mathscr{R} \nabla g(u)]_{D}\right)
$$

for any $f, g \in \mathscr{F}_{\widetilde{\mathbb{A}}}(u)$ are closed. Moreover, the corresponding Hamiltonian operators, determined from the relationships (3.19) and (3.20) via the identifications

$$
\omega_{1}^{(2)}\left(\xi_{f}, \xi_{g}\right):=\left(\vartheta_{1} \nabla f, \nabla g\right), \quad \omega_{2}^{(2)}\left(\xi_{f}, \xi_{g}\right):=\left(\vartheta_{2} \nabla f, \nabla g\right),
$$

are compatible; that $i$, s the sum $\lambda \vartheta_{1}+\mu \vartheta_{2}: \mathscr{L}_{\widetilde{\mathbb{A}}} \rightarrow \mathscr{L}_{\widetilde{\mathbb{A}}}$ for arbitrary $\lambda, \mu \in \mathbb{K}$ is also a Hamiltonian operator.

Proof. (Sketch). As the 2-form (3.19) is closed a priori, a proof of the proposition consists in directly checking the closedness of the 2-forms $\omega_{2}^{(2)} \in \Lambda^{2}(\widetilde{\mathbb{A}}(u))$, which is equivalent to the YangBaxter condition (3.18). Taking into account the relationships (3.19) and (3.20) and the representation of the Hamiltonian operator $\vartheta_{2}: \mathscr{L}_{\widetilde{\mathbb{A}}} \rightarrow \mathscr{L}_{\widetilde{\mathbb{A}}}$ as the composition $\vartheta_{2}=\vartheta_{1}^{-1} \vartheta_{0}$, where the Hamiltonian operator $\vartheta_{0}: \mathscr{L}_{\widetilde{\mathbb{A}}} \rightarrow \mathscr{L}_{\widetilde{\mathbb{A}}}^{*}$ is naturally determined from the canonical Lie-Poisson bracket (3.4) as

$$
\left(u,[\nabla f(u), \nabla g(u)):=\left(\vartheta_{0} \nabla f(u), \nabla g(u)\right)\right.
$$

for any $f, g \in \mathscr{F}_{\widetilde{\mathbb{A}}}(u)$, the representation $\vartheta_{2}=\vartheta_{1}^{-1} \vartheta_{0}$ is exactly equivalent $[18,20,41,59,76]$ to the compatibility of the Hamiltonian operators $\vartheta_{1}$ and $\vartheta_{2}$ on $\widetilde{\mathbb{A}}(u)$. We note here that if the parameter $\alpha=0$, the expression (3.19) determines a 2-cocycle on the Lie algebra $\mathscr{L}_{\widetilde{\mathbb{A}}}$ owing to the fact that the inverse mapping $\mathscr{R}^{-1}: \mathscr{L}_{\widetilde{\mathbb{A}}} \rightarrow \mathscr{L}_{\widetilde{\mathbb{A}}}$ is a weak differentiation on $\mathscr{L}_{\widetilde{\mathbb{A}}}$, that is

$$
\left(c, \mathscr{R}^{-1}[a, b]_{D}\right)=\left(c,\left[\mathscr{R}^{-1} a, b\right]_{D}+\left[a, \mathscr{R}^{-1} b\right]_{D}\right)
$$

for any $a, b$ and $c \in \mathscr{L}_{\widetilde{\mathbb{A}}}$. As a consequence one immediately infers that the operator $\lambda \vartheta_{0}+\mu \vartheta_{1}$ : $\mathscr{L}_{\widetilde{\mathbb{A}}} \rightarrow \mathscr{L}_{\widetilde{\mathbb{A}}}$ for arbitrary $\lambda, \mu \in \mathbb{K}$ is Hamiltonian, which implies the compatibility of the Hamiltonian operators $\vartheta_{1}$ and $\vartheta_{2}$ on $\widetilde{\mathbb{A}}(u)^{*}$.

The $\mathscr{R}$-structures on the weak Lie algebra $\mathscr{L}_{\widetilde{\mathbb{A}}}$ can be effectively exploited for constructing additional Hamiltonian operators on $\mathscr{L}_{\widetilde{\mathbb{A}}}^{*}$ owing to the fact that the bracket

$$
\left(c,[a, b]_{(\mathscr{R})}\right):=\left(c,[a, \mathscr{R} b]_{D}+[\mathscr{R} a, b]_{D}\right)
$$

generates for any $a, b$ and $c \in \mathscr{L}_{\widetilde{\mathbb{A}}}$ a new weak Lie structure on the linear space $\mathscr{L}_{\widetilde{\mathbb{A}}}$, thus producing a new weak Lie algebra $\mathscr{L}_{\widetilde{\mathbb{A}}}^{(\mathscr{R})}:=\left(\widetilde{\mathbb{A}},+,[\cdot, \cdot]_{(\mathscr{R})}\right)$. 
Example 3.2. Consider the endomorphism $\mathscr{R}: \mathscr{L}_{\widetilde{\mathbb{A}}} \rightarrow \mathscr{L}_{\widetilde{\mathbb{A}}}$, where for any $a \in \mathscr{L}_{\widetilde{\mathbb{A}}}$ we put

$$
\mathscr{R}(a):=\frac{1}{2}\left[\int_{0}^{x} a(y) d y-\int_{x}^{2 \pi} a(y) d y\right]
$$

for any $x \in \mathbb{S}^{1}$, which satisfies the weak Yang-Baxter commutator condition (3.15). Then it is easy to check that the inverse mapping $\mathscr{R}^{-1}=d / d x, \quad x \in \mathbb{S}^{1}$, is the natural skew-symmetric differentiation of the weak Lie algebra $\mathscr{L}_{\widetilde{\mathbb{A}}}$, generating a Poisson structure compatible with that of (3.12).

In a manner similar to the above $[51,59,75,76]$, one verifies the existence of the following so called "quadratic" Poisson brackets. Namely, the next proposition holds.

Proposition 3.4. Let the weak Yang-Baxter condition (3.18) hold. Then the brackets

$$
\{f, g\}_{1}:=(u \circ \nabla f(u), \mathscr{R}(u \circ \nabla g(u)))-(\nabla f(u) \circ u, \mathscr{R}(\nabla g(u) \circ u))
$$

and

$$
\{f, g\}_{2}:=\left(u,[\mathscr{R} \nabla f(u), \nabla g(u)]_{D}+[\nabla f(u), \mathscr{R} \nabla g(u)]_{D}\right),
$$

defined for any $f, g \in \mathscr{F}_{\widetilde{\mathbb{A}}}(u)$, are Poisson and compatible on $\widetilde{\mathbb{A}}(u)$.

As an interesting and also useful consequence of the $\mathscr{R}$-matrix construction, one has the fact that the following subspaces

$$
\mathscr{L}_{\widetilde{\mathbb{A}}}^{ \pm}:=(\mathbb{I} \pm \mathscr{R}) / 2 \in \mathscr{L}_{\widetilde{\mathbb{A}}}
$$

are Lie sub-algebras of $\mathscr{L}_{\widetilde{\mathbb{A}}}$, which is equivalent to the condition that the mappings

$$
(\mathbb{I} \pm \mathscr{R}) / 2: \mathscr{L}_{\widetilde{\mathbb{A}}}^{(\mathscr{R})} \rightarrow \mathscr{L}_{\widetilde{\mathbb{A}}}^{ \pm} \subset \mathscr{L}_{\widetilde{\mathbb{A}}}
$$

are homomorphisms [76] of the Lie algebras $\mathscr{L}_{\widetilde{\mathbb{A}}}^{(\mathscr{R})}:=\left(\widetilde{\mathbb{A}},[\cdot, \cdot]_{(\mathscr{R})}\right)$ and $\mathscr{L}_{\widetilde{\mathbb{A}}}^{ \pm}$. In the special case when $\mathscr{L}_{\widetilde{\mathbb{A}}}^{+} \cap \mathscr{L}_{\widetilde{\mathbb{A}}}^{-}=\{0\}$, the operator $\mathscr{R}=P_{+}-P_{-}$, the linear operators $P_{ \pm}:=(\mathbb{I} \pm \mathscr{R}) / 2: \mathscr{L}_{\widetilde{\mathbb{A}}} \rightarrow \mathscr{L}_{\widetilde{\mathbb{A}}}$ are projectors and the weak Lie algebra $\mathscr{L}_{\widetilde{\mathbb{A}}}$ allows the direct sum splitting $\mathscr{L}_{\widetilde{\mathbb{A}}}=\mathscr{L}_{\widetilde{\mathbb{A}}}^{+} \oplus \mathscr{L}_{\widetilde{\mathbb{A}}}^{-}$.

\section{Integrable Riemann hydrodynamical systems and related multicomponent Hamiltonian operators}

As the expressions (2.30), (2.36) and (2.40) are true Hamiltonian operators on the functional space $\mathscr{F}_{\widetilde{\mathbb{A}}}(u)$ satisfying the Schouten-Nijenhuis condition (2.10), following the algebraic scheme of [78] mentioned above and using the results of [67] and the right Leibniz algebra (2.35) and the new Riemann algebra (2.39), one can describe a wide class of multicomponent completely integrable dynamical systems containing the infinite hierarchies of multicomponent Riemann hydrodynamical flows. For instance, consider the generalized completely integrable Riemann type dynamical system

$$
D_{t} u_{1}=u_{2}, D_{t} u_{2}=u_{3}, \ldots, D_{t} u_{N}=0
$$

on the functional space $\widetilde{\mathbb{A}}(u)$ for some non-associative and non-commutative finite-dimensional algebra $\mathbb{A}$, where $D_{t}:=\partial / \partial t+u_{1} D_{x}, D_{x}:=\partial / \partial x, x \in \mathbb{S}^{1}$ and $N \in \mathbb{Z}_{+}$, which was recently studied in detail in $[66,67]$. The relationships (2.39) allow to calculate the corresponding representations 
of the Riemann algebra $\mathbb{A}$ for cases $N=2$ and $N=3$, giving rise to the corresponding Hamiltonian operators $\vartheta(u): \mathscr{L}_{\widetilde{\mathbb{A}}} \rightarrow \mathscr{L}_{\widetilde{\mathbb{A}}}$, coinciding with those constructed in [67] modulo the trivial constant 2 -cocycles on the weak adjacent loop Lie algebra $\mathscr{L}_{\widetilde{\mathbb{A}}}$. In fact, for the case $N=2$ one easily obtains from (2.40) the skew-symmetric two-dimensional matrix differentiation representation

$$
\vartheta_{2}(u):=\left(\begin{array}{cc}
0 & u_{1, x} D_{x}^{-1} \\
D_{x}^{-1} u_{1, x} & u_{2, x} D_{x}^{-1}+D_{x}^{-1} u_{2, x}
\end{array}\right),
$$

coinciding, modulo the trivial constant 2-cocycle

$$
\varpi_{2}(a, b):=f_{2}\left(D_{x}^{-1} a, b\right),
$$

determined for any $a, b \in \mathscr{L}_{\widetilde{\mathbb{A}}}$ and a suitable symmetric bilinear form $f_{2}: \mathscr{L}_{\widetilde{\mathbb{A}}} \times \mathscr{L}_{\widetilde{\mathbb{A}}} \rightarrow \mathbb{K}$, with the Hamiltonian operator

$$
\eta_{2}(u)=\left(\begin{array}{cc}
D_{x}^{-1} & u_{1, x} D_{x}^{-1} \\
D_{x}^{-1} u_{1, x} & u_{2, x} D_{x}^{-1}+D_{x}^{-1} u_{2, x}
\end{array}\right)
$$

on the space $\mathscr{F}_{\widetilde{\mathbb{A}}}(u)$ for the Riemann type dynamical system (4.1), whose Hamiltonian representation

$$
\frac{d}{d t}\left(u_{1}, u_{2}\right)^{\top}=-\eta_{2}(u) \nabla H_{2}\left(u_{1}, u_{2}\right)
$$

holds for the Hamiltonian function $H_{2} \in \mathscr{F}_{\widetilde{\mathbb{A}}}(u)$, equal to

$$
H_{2}:=\frac{1}{2} \int_{0}^{2 \pi}\left(u_{2} u_{1, x}-u_{1} u_{2, x}\right) d x
$$

Proceeding similarly for the case $N=3$, one easily obtains from (2.40) the skew-symmetric three-dimensional matrix Hamiltonian operator $\vartheta_{3}(u): \mathscr{L}_{\widetilde{\mathbb{A}}} \rightarrow \mathscr{L}_{\widetilde{\mathbb{A}}}$ representation

$$
\vartheta_{3}(u)=\left(\begin{array}{ccc}
0 & u_{1, x} D_{x}^{-1} & 0 \\
D_{x}^{-1} u_{1, x} & u_{2, x} D_{x}^{-1}+D_{x}^{-1} u_{2, x} & D_{x}^{-1} u_{3, x} \\
0 & u_{3, x} D_{x}^{-1} & 0
\end{array}\right),
$$

coinciding, modulo the trivial constant 2-cocycle

$$
\varpi_{2}(a, b):=f_{3}\left(D_{x}^{-1} a, b\right),
$$

determined for any $a, b \in \mathscr{L}_{\widetilde{\mathbb{A}}}$ and a suitable symmetric bilinear form $f_{3}: \widetilde{\mathbb{A}} \times \widetilde{\mathbb{A}} \rightarrow \mathbb{K}$ with the Hamiltonian operator

$$
\eta_{3}(u)=\left(\begin{array}{ccc}
D_{x}^{-1} & u_{1, x} D_{x}^{-1} & 0 \\
D_{x}^{-1} u_{1, x} & u_{2, x} D_{x}^{-1}+D_{x}^{-1} u_{2, x} & D_{x}^{-1} u_{3, x} \\
0 & u_{3, x} D_{x}^{-1} & 0
\end{array}\right)
$$

on the functional space $\mathscr{F}_{\widetilde{\mathbb{A}}}(u)$ for the Riemann dynamical system (4.1), whose Hamiltonian representation

$$
\frac{d}{d t}\left(u_{1}, u_{2}, u_{3}\right)^{\top}=-\eta_{3}(u) \nabla H_{3}\left[u_{1}, u_{2}, u_{3}\right]
$$

holds for a suitably constructed Hamiltonian function $H_{3} \in \mathscr{F}_{\widetilde{\mathbb{A}}}(u)$. 
There is also an interesting observation concerning an infinite hierarchy [67] of the generalized Riemann hydrodynamic systems

$$
D_{t} u_{1}=u_{2}, D_{t} u_{2}=u_{3}, \ldots, D_{t} u_{N-1}=\left(\bar{u}_{N, x}\right)^{s}, D_{t} \bar{u}_{N}=0
$$

on the functional space $\mathscr{F}_{\widetilde{\mathbb{A}}}(u)$, where $s, N \in \mathbb{Z}_{+}$, with the algebra $\mathbb{A}$ generated by the constraints (2.39). For the case $s=2$ and $N=3$ the above skew-symmetric three-dimensional matrix Hamiltonian operator $\bar{\vartheta}_{3 \mid 2}(u): \mathscr{L}_{\widetilde{\mathbb{A}}} \rightarrow \mathscr{L}_{\widetilde{\mathbb{A}}}$ representation

$$
\bar{\vartheta}_{3 \mid 2}(u)=\left(\begin{array}{ccc}
0 & u_{1, x} D_{x}^{-1} & 0 \\
D_{x}^{-1} u_{1, x} & u_{2, x} D_{x}^{-1}+D_{x}^{-1} & u_{2, x} \\
0 & \bar{u}_{3, x}^{-1} D_{x}^{-1} & 0
\end{array}\right)
$$

proves to coincide, modulo the trivial constant 2-cocycle

$$
\bar{\omega}_{2, \bar{\eta}}(a, b):=f_{\bar{\eta}}\left(D_{x}^{-1} a, b\right),
$$

determined for any $a, b \in \mathscr{L}_{\widetilde{\mathbb{A}}}$ and a suitable symmetric bilinear form $f_{\bar{\eta}}: \mathscr{L}_{\widetilde{\mathbb{A}}} \times \mathscr{L}_{\widetilde{\mathbb{A}}} \rightarrow \mathbb{K}$, exactly with the Hamiltonian operator

$$
\bar{\eta}_{3 \mid 2}(u)=\left(\begin{array}{ccc}
D_{x}^{-1} & u_{1, x} D_{x}^{-1} & 0 \\
D_{x}^{-1} u_{1, x} & u_{2, x} D_{x}^{-1}+D_{x}^{-1} u_{2, x} & D_{x}^{-1} \bar{u}_{3, x} \\
0 & \bar{u}_{3, x} D_{x}^{-1} & 0
\end{array}\right)
$$

on the functional space $\mathscr{F}_{\widetilde{\mathbb{A}}}(u)$ for the Riemann type dynamical system (4.11), whose Hamiltonian representation

$$
\frac{d}{d t}\left(u_{1}, u_{2}, \bar{u}_{3}\right)^{\top}=-\bar{\eta}_{3 \mid 2}(u) \nabla H_{3 \mid 2}\left(u_{1}, u_{2}, \bar{u}_{3}\right)
$$

holds for the Hamiltonian function $H_{3 \mid 2} \in \mathscr{F}_{\widetilde{\mathbb{A}}}(u)$, equal to

$$
H_{3 \mid 2}:=\frac{1}{2} \int_{0}^{2 \pi}\left[2 u_{1}\left(\bar{u}_{3, x}\right)^{2}-u_{2}^{2}-u_{1}^{2} u_{2, x}\right] d x .
$$

Moreover, one can calculate such a constant 2-cocycle on the Lie algebra $\mathscr{L}_{\widetilde{\mathbb{A}}}$

$$
\varpi_{2, \bar{\vartheta}}(a, b):=f_{\bar{\vartheta}}^{(1)}\left(D_{x}^{-1} a, b\right)+f_{\bar{\vartheta}}^{(2)}(a, b),
$$

determined for any $a, b \in \mathscr{L}_{\widetilde{\mathbb{A}}}$ by means of two suitably symmetric $f_{\bar{\vartheta}}^{(1)}: \mathscr{L}_{\widetilde{\mathbb{A}}} \times \mathscr{L}_{\widetilde{\mathbb{A}}} \rightarrow \mathbb{K}$ and skewsymmetric $f_{\bar{\vartheta}}^{(2)}: \mathscr{L}_{\widetilde{\mathbb{A}}} \times \mathscr{L}_{\widetilde{\mathbb{A}}} \rightarrow \mathbb{K}$ bilinear forms, which naturally generates the (4.14) - compatible Hamiltonian operator

$$
\bar{\vartheta}_{3 \mid 2}^{(0)}(u)=\left(\begin{array}{ccc}
0 & 1 & 0 \\
-1 & 0 & 0 \\
0 & 0 & 1 / 2 D_{x}^{-1}
\end{array}\right)
$$

on the functional space $\mathscr{F}_{\widetilde{\mathbb{A}}}(u)$ for (4.11), whose Hamiltonian representation

$$
\frac{d}{d t}\left(u_{1}, u_{2}, \bar{u}_{3}\right)^{\top}=-\bar{\vartheta}_{3 \mid 2}^{(0)}(u) \nabla H_{3 \mid 2}^{(0)}\left(u_{1}, u_{2}, \bar{u}_{3}\right)
$$


holds for the Hamiltonian function $H_{3 \mid 2}^{(0)} \in \mathscr{F}_{\widetilde{\mathbb{A}}}(u)$, equal to

$$
H_{3 \mid 2}^{(0)}:=\frac{1}{2} \int_{0}^{2 \pi}\left[u_{1} u_{2, x}-u_{2} u_{1, x}-2\left(\bar{u}_{3, x}\right)^{2}\right] d x .
$$

It is worth noting here, as was already remarked in [68], that the generalized Riemann hydrodynamic system (4.11) for $s=3, N=3$ reduces to the well-known integrable Degasperis-Processi dynamical system $[30,31]$ for the function $u:=u_{1}$ :

$$
u_{t}-u_{x x t}+4 u u_{x}-3 u_{x} u_{x x}-u u_{x x x}=0 .
$$

Also, for $s=2, N=3$, the system (4.11) for the function $u:=u_{1}$ reduces to the well-known [28] integrable Camassa-Holm dynamical system

$$
u_{t}-u_{x x t}+3 u u_{x}-2 u_{x} u_{x x}+u u_{x x x}=0,
$$

whose multicomponent extensions were recently extensively studied in $[29,37,44,78]$.

Now, returning to the case $N=2$ of the system (4.1), it reduces under the substitutions $u_{1}:=u$, $u_{2}:=D_{x}^{-1} u_{x}^{2} / 2$ to the well-known $[42,45,63,70]$ Hunter-Saxton nonlinear dynamical system

$$
d u / d t=-u u_{x}+D_{x}^{-1} u_{x}^{2} / 2
$$

on the functional manifold $\widetilde{\mathbb{K}}(u), u \in \widetilde{\mathbb{K}}^{*}$, describing propagation of short-waves in a relaxing medium with spatial memory effects. As as shown in [63,65,70], the dynamical system (4.22) is a completely integrable bi-Hamiltonian flow on the functional manifold $\widetilde{\mathbb{K}}(u), u \in \widetilde{\mathbb{K}}^{*}$, with respect to the compatible pair of scalar Hamiltonian operators $\vartheta_{1}(u), \vartheta_{2}(u): T^{*}(\widetilde{\mathbb{K}}(u)) \rightarrow T(\widetilde{\mathbb{K}}(u))$ :

$$
\vartheta_{1}(u)=D_{x}^{-1}, \quad \vartheta_{2}(u)=u D_{x}^{-1}+D_{x}^{-1} u .
$$

As we are interested in the corresponding multicomponent generalization of the dynamical system (4.22), we need to consider the functional space $\widetilde{\mathbb{A}}(u), u \in \widetilde{\mathbb{A}}^{*}$, generated by a finite-dimensional noncommutative and non-associative algebra $\mathbb{A}$, and construct the Poisson operators on $\mathscr{F}_{\widetilde{A}}(u)$ in the form (2.36), related to the right Leibniz algebra structure (2.35) and reducing at $N=1$ to the scalar Hamiltonian operator $\vartheta_{2}(u): T^{*}(\widetilde{\mathbb{K}}(u)) \rightarrow T(\widetilde{\mathbb{K}}(u))$ from the pair (4.23). Moreover, as the compatible Hamiltonian operators are generated by means of suitable central extensions of the adjacent weak Lie algebra, the problem of description them, as was noted above, requires a detailed investigation of the structural properties and finite-dimensional representations of the right Leibniz algebra defined by the constraints (2.35). The next Section is devoted to their preliminary algebraic studying.

\section{Leibniz algebra structure}

\subsection{General setting}

In preceding sections we have shown that any skew-symmetric Lie structure $[\cdot, \cdot]_{D}$ on the weak adjacent Lie algebra $\mathscr{L}_{\widetilde{\mathbb{A}}}$, satisfying the Jacobi identity and nontrivially depending on the differentiation $D_{x}: \widetilde{\mathbb{A}} \rightarrow \widetilde{\mathbb{A}}$, determines on the phase space $\mathscr{F}_{\widetilde{\mathbb{A}}}(u)$ the local Poisson bracket

$$
\{f, g\}(u):=\prec u,[\nabla f(u(x)), \nabla g(u(x)))]_{D} \succ
$$

for any $u \in \widetilde{\mathbb{A}}^{*}$ and arbitrary functions $f, g \in \mathscr{F}_{\widetilde{\mathbb{A}}}(u)$. Moreover, from the analysis provided above we know that if the Hamiltonian operator $\vartheta(u): \mathscr{L}_{\widetilde{\mathbb{A}}} \rightarrow \mathscr{L}_{\widetilde{\mathbb{A}}}^{*} \simeq \mathscr{L}_{\widetilde{\mathbb{A}}}$ related to (2.29) corresponds 
to some 2-cocycle on the Lie algebra $\mathscr{L}_{\widetilde{\mathbb{A}}}$, then it will be a priori Hamiltonian. Moreover, owing to Proposition 3.1 , if this 2-cocycle is generated by a differentiation $\mathrm{D}_{u}: \mathscr{L}_{\widetilde{\mathbb{A}}} \rightarrow \mathscr{L}_{\widetilde{\mathbb{A}}}, u \in \widetilde{\mathbb{A}}^{*} \simeq \widetilde{\mathbb{A}}$, on the Lie algebra $\mathscr{L}_{\widetilde{\mathbb{A}}}$, one need only check the related weak Leibniz property (3.8) in the weak adjacent Lie algebra $\mathscr{L}_{\widetilde{\mathbb{A}}}$. In Section 2 we already showed that the skew-symmetric structure

$$
[a, b]_{D}=D_{x} a \circ b-D_{x} b \circ a
$$

for any $a, b \in \mathscr{L}_{\widetilde{\mathbb{A}}}$, imposed on the weak adjacent Lie algebra $\mathscr{L}_{\widetilde{\mathbb{A}}}$, gives rise to the Hamiltonian operator (2.30) on $\widetilde{\mathbb{A}}(u)$

$$
\vartheta(u)=\sigma(u) D_{x}+D_{x} \sigma(u)^{\top}
$$

and to the related multiplicative Novikov algebra structure $(2.32)$ on $\mathbb{A}$

$$
\left[R_{a}, R_{b}\right]=0,\left[L_{a}, L_{b}\right]=L_{[a, b]}
$$

for any $a, b$ and $c \in \mathbb{A}$. Similarly, the skew-symmetric structure (2.34)

$$
[a, b]_{D}=D_{x}^{-1} a \circ b-D_{x}^{-1} b \circ a
$$

for any $a, b \in \mathscr{L}_{\widetilde{\mathbb{A}}}$ on the weak adjacent Lie algebra $\mathscr{L}_{\widetilde{\mathbb{A}}}$ gives rise to the Hamiltonian operator (2.30) on $\widetilde{\mathbb{A}}(u)$

$$
\vartheta(u)=\sigma(u) D_{x}^{-1}+D_{x}^{-1} \sigma(u)^{\top}
$$

and to the related multiplicative right Leibniz algebra structure (2.32) on $\mathbb{A}$

$$
R_{a \circ b}=\left[R_{a}, R_{b}\right], \quad R_{a \circ b}+R_{b \circ a}=0 .
$$

Moreover, the skew-symmetric structure (2.34)

$$
[a, b]_{D}=D_{x}^{-1} b \circ D_{x} a-D_{x}^{-1} a \circ D_{x} b
$$

for any $a, b \in \mathscr{L}_{\widetilde{\mathbb{A}}}$ on the weak adjacent Lie algebra $\mathscr{L}_{\widetilde{\mathbb{A}}}$ gives rise to the Hamiltonian operator (2.38) on $\widetilde{\mathbb{A}}(u)$

$$
\vartheta(u)=D_{x} \sigma(u) D_{x}^{-1}-D_{x}^{-1} \sigma(u)^{\top} D_{x}
$$

and to the related multiplicative Riemann algebra structure (2.39) on $\mathbb{A}$

$$
\left[R_{a}, R_{b}\right]=0, L_{a \circ b}=R_{a \circ b}=L_{b \circ a}
$$

for any $a, b$ and $c \in \mathbb{A}$.

Remark 5.1. Just as in Section 2 , one can construct for any $a, b$ and $c \in \mathbb{A}$ dual Novikov 


$$
R_{[a, b]}=\left[R_{b}, R_{a}\right], \quad\left[L_{a}, L_{b}\right]=0
$$

Leibniz

$$
L_{a \circ b}=\left[L_{a}, L_{b}\right], \quad L_{a \circ b}+L_{b \circ a}=0
$$

and Riemann

$$
\left[L_{a}, L_{b}\right]=0, R_{a \circ b}=L_{a \circ b}=R_{b \circ a}
$$

algebra constraints, respectively related to the adjacent Lie algebra $\mathscr{L}_{\widetilde{\mathbb{A}}}$ structures

$$
\begin{aligned}
& {[a, b]_{D}=D_{x} a \circ b-D_{x} b \circ a,} \\
& {[a, b]_{D}=a \circ D_{x}^{-1} b-b \circ D_{x}^{-1} a}
\end{aligned}
$$

and

$$
[a, b]_{D}=-D_{x} a \circ D_{x}^{-1} b+D_{x} b \circ D_{x}^{-1} a
$$

for any $a, b \in \mathscr{L}_{\widetilde{\mathbb{A}}}$.

As mentioned above, simultaneously we have shown that the expressions (5.3), (5.7) and (5.9) are true Hamiltonian operators on the functional space $\mathscr{F}_{\widetilde{\mathbb{A}}}(u)$ satisfying the Schouten-Nijenhuis condition (2.10). Using the algebraic scheme in [78] and the right Leibniz algebra (5.7) and the new Riemann algebra (5.9), one can describe a wide class of multicomponent completely integrable dynamical systems containing, as follows from the recent results in [67], infinite hierarchies of the multicomponent hydrodynamical Riemann type systems.

\subsection{Preliminaries}

An algebra $(\mathbb{A},+, \cdot)$ over a field $\mathbb{K}$ is called a (right) Leibniz algebra if it satisfies the identity

$$
x(y z)=(x y) z-(x z) y
$$

for any $x, y, z \in \mathbb{A}$. Any Lie algebra is clearly a Leibniz algebra. Leibniz algebras were introduced by A.M. Bloh [21,22] and rediscovered by J.-L. Loday [53]. By analogy, we can introduce the notion of a (right) Leibniz ring. Recall that a subalgebra (respectively a subring) $H \subseteq \mathbb{A}$ is said to be an ideal of a Leibniz algebra (respectively a Leibniz ring) $\mathbb{A}$ if $H \cdot \mathbb{A}, \mathbb{A} \cdot H \subseteq H$. If an additive subgroup $S^{+}$is of finite index in the additive group $\mathbb{A}^{+}$of a Leibniz algebra (respectively a Leibniz ring) $\mathbb{A}$, then we say that $S$ is of finite index in $\mathbb{A}$, i.e. $|\mathbb{A}: S|<\infty$.

A linear mapping $\delta: \mathbb{A} \rightarrow \mathbb{A}$ is called a derivation of $\mathbb{A}$ if

$$
\delta(x y)=\delta(x) y+x \delta(y)
$$

for all $x, y \in \mathbb{A}$. The set Der $\mathbb{A}$ of all derivations of a Leibniz algebra $\mathbb{A}$ is a Lie algebra with respect to operations addition " + ". A linear mapping $F: \mathbb{A} \rightarrow \mathbb{A}$ is called a generalized derivation of a Leibniz algebra $\mathbb{A}$ associated with a derivation $\delta \in \operatorname{Der} \mathbb{A}$ (in the sense of Brešar [24]) if

$$
F(x y)=F(x) y+x \delta(y)
$$

for any $x, y \in \mathbb{A}$. The set of all generalized derivations of $\mathbb{A}$ we denote by GDer $\mathbb{A}$. We will write $(F, \delta) \in \operatorname{GDer} \mathbb{A}$ iff $F$ is a generalized derivation of $\mathbb{A}$ associated with $\delta \in \operatorname{Der} \mathbb{A}$. Since $(\delta, \delta) \in$ 
GDer $\mathbb{A}$ for any $\delta \in \operatorname{Der} \mathbb{A}$, we conclude that

$$
\operatorname{Inn} \mathbb{A} \subseteq \operatorname{Der} \mathbb{A} \subseteq \text { GDer } \mathbb{A} .
$$

A generalized derivation $F$ of $\mathbb{A}$ that is associated with an inner derivation $r_{a} \in \operatorname{Inn} \mathbb{A}$ is called $a$ generalized inner derivation of $\mathbb{A}$. By IGDer $\mathbb{A}$ we denote the set of all generalized inner derivations of $\mathbb{A}$. Another of the various generalizations of Lie (and Leibniz) algebra derivations was introduced in $[14,27,39,49,55]$ and elsewhere.

If $\mathbb{A}$ is a Leibniz algebra, then

$$
\operatorname{Leib}(\mathbb{A}):=\operatorname{span}\left\{x^{2} \mid x \in \mathbb{A}\right\}
$$

is the smallest ideal of $\mathbb{A}$ such that the quotient algebra $\mathbb{A} / \operatorname{Leib}(\mathbb{A})$ is a Lie algebra (see e.g. $[14,32]$ ). The center

$$
Z(\mathbb{A})=\{u \in \mathbb{A} \mid a \mathbb{A}=0=\mathbb{A} u\}
$$

and the right annihilator

$$
\operatorname{rann} \mathbb{A}:=\{t \in \mathbb{A} \mid \mathbb{A} t=0\}
$$

of $\mathbb{A}$ are its ideals such that $Z(\mathbb{A}) \subseteq \operatorname{Leib}(\mathbb{A}) \subseteq \operatorname{rann} \mathbb{A}$. The left annihilator

$$
\operatorname{lann} \mathbb{A}:=\{t \in \mathbb{A} \mid t \mathbb{A}=0\}
$$

of $\mathbb{A}$ is its right ideal.

Remark 5.2. It is known that a simple Lie ring is perfect, but there exist non-complete simple Lie algebras (see [54]).

Now, let $D=\operatorname{Der} \mathbb{A}, G=$ GDer $\mathbb{A}, \Delta$ be a non-empty subset of $D$ (respectively $G$ ). If $I$ is an ideal of $\mathbb{A}$ and $\delta(I) \subseteq I$ for all $\delta \in \Delta$, then $I$ is called a $\Delta$-ideal of $\mathbb{A}$. Inasmuch as $(x+d(x))(x+d(x)) \in$ $\operatorname{Leib}(\mathbb{A})$ for any $x \in \mathbb{A}$ and $d \in D$, we deduce that $d\left(x^{2}\right) \in \operatorname{Leib}(\mathbb{A})$ and so $\operatorname{Leib}(\mathbb{A})$ is a $D$-ideal of $\mathbb{A}$. For a Leibniz algebra $(\mathbb{A},+, \cdot)$, define the derived sequence as follows:

$$
\mathbb{A}^{(1)}=\mathbb{A}, \mathbb{A}^{(2)}=\mathbb{A} \mathbb{A}, \mathbb{A}^{(k+1)}=\mathbb{A}^{(k)} \mathbb{A} \quad(k \geq 1) .
$$

A Leibniz algebra $\mathbb{A}$ is called nilpotent if there exists a positive integer $s$ such that $\mathbb{A}^{(s)}=0$ (see e.g. $[2,32,39,74])$. Moreover, $\mathbb{A}^{(2)}$ is called the commutator ideal of $\mathbb{A}$.

Let $X \in\{0, D, G\}$. An ideal $Y$ of a (Lie or Leibniz) algebra $\mathbb{A}$ is called:

- $X$-semisimple if, for any $X$-ideal $T$ of $\mathbb{A}$, the condition $T^{2} \subseteq Y$ implies that $T \subseteq Y$,

- $X$-prime if, for any $X$-ideals $T, Q$ of $\mathbb{A}$, the condition $T Q \subseteq Y$ implies that $T \subseteq Y$ or $Q \subseteq Y$,

- $X$-simple if $[\mathbb{A}, \mathbb{A}] \neq Y$ and it only has the following $X$-ideals: $0, Y$ and $\mathbb{A}$ (here 0 and $Y$ are not necessarily different),

- $X$-primary if, for any $X$-ideals $T, Q$ of $\mathbb{A}$, the condition $T Q \subseteq Y$ implies that $T \subseteq Y$ or $Q^{(m)} \subseteq Y$ for some positive integer $m$.

In particular, a $X$-semisimple (respectively $X$-prime, $X$-simple or $X$-primary) ideal $Y$ is called semisimple (respectively prime, simple or primary) if $X=0$. The quotient algebra $\mathbb{A} / Y$ is semisimple (respectively prime or primary) if and only if the ideal $Y$ is the ones. A Leibniz algebra $\mathbb{A}$ is 
semisimple (respectively prime, primary) if and only if the Lie algebra $\mathbb{A} / \operatorname{Leib}(\mathbb{A})$ is the ones. If $\mathbb{A}$ is a simple Leibniz algebra, then $\mathbb{A} / \operatorname{Leib}(\mathbb{A})$ is a simple Lie algebra, but the opposite is not true. It is not difficult to check that if a Leibniz algebra $\mathbb{A}$ is prime (respectively semisimple or simple), then $\operatorname{rann} \mathbb{A}=\operatorname{Leib}(\mathbb{A})$. Every semisimple (respectively prime, simple or primary) Leibniz algebra is $D$-semisimple (respectively $D$-prime, $D$-simple or $D$-primary).

Many authors have investigated derivations of Leibniz algebras in the context of geometric studies of algebras (see, e.g. [48, 58, 71-73]) and representations of Leibniz algebras (see e.g. [39, 40, 54, 55]). For example, A. Fialowski, A.Kh. Khudoyberdiyev and B.A. Omirov [39] have proved that a Leibniz algebra is nilpotent iff it admits an invertible Leibniz-derivation, B.A. Omirov [58] , I.S. Rakhimov and A.-H. Al-Nashi [73] have studied derivation algebras of filiform Leibniz algebras, M. Ladra, I.M. Rikhsiboev and R.M. Turdibaev [48] proved that a finite-dimensional Leibniz algebra $\mathbb{A}$ with a nonsingular derivation is nilpotent, I.S. Rakhimov, K.K. Masutova and B.A. Omirov [71] proved, in particular, that any derivation of a simple finite-dimensional Leibniz algebra over a field of zero characteristic can be represented as sum of three derivations of special form. In this paper we study connections between Leibniz algebras $\mathbb{A}$, their derivation algebras $\operatorname{Der} \mathbb{A}$ and generalized derivation algebras GDer $\mathbb{A}$. Our first result in this direction is the following.

Proposition 5.1. Let $\mathbb{A}$ be a Leibniz algebra. Then the following hold:

(1) $D$ is a simple Lie algebra iff $\mathbb{A}$ is a simple Leibniz algebra and $D=\operatorname{Inn} \mathbb{A}=[D, D]$,

(2) If $D$ is a prime (respectively semisimple or primary) Lie algebra, then $\mathbb{A}$ is a D-prime (respectively D-semisimple or D-primary) Leibniz algebra.

Obviously, a finite-dimensional Leibniz algebra $\mathbb{A}$ is semisimple if its maximal solvable ideal is equal to $\operatorname{Leib}(\mathbb{A})$. Semisimple Leibniz algebras have been studied in $[2,32,43,71]$ and elsewhere. In this way we will state the next result.

Theorem 5.1. If $\mathbb{A}$ is a D-prime (respectively D-semisimple) Leibniz algebra, then $D / A D e r \mathbb{A}$ is prime (respectively semisimple) Lie algebra, where $\operatorname{ADer} \mathbb{A}:=\{\delta \in \operatorname{Der} \mathbb{A} \mid \delta(\mathbb{A}) \subseteq \operatorname{rann} \mathbb{A}\}$.

We also prove the following property.

Theorem 5.2. Let $\mathbb{A}$ be a Leibniz algebra (respectively a Leibniz ring). Then the following statements are equivalent:

(1) $|\mathbb{A}: \operatorname{rann} \mathbb{A}|<\infty$,

(2) $\operatorname{Inn} \mathbb{A}$ is finite.

In the proof of this theorem we use the fact that if $S$ is a subalgebra (respectively a subring) of finite index in a Leibniz algebra (respectively a Leibniz ring) $\mathbb{A}$, then $\mathbb{A}$ has an ideal $I$ of finite index such that $I \subseteq S$ (Proposition 5.2).

For basic definitions and properties of Leibniz and Lie algebras we refer the reader to $[2,5,46$, 53].

\subsection{Properties of derivation algebras}

We first give some information about derivation algebras. If $A \subseteq \mathbb{A}$, then

$$
\operatorname{Inn}_{A} \mathbb{A}:=\left\{r_{a} \mid a \in A\right\}
$$

and, in particular, $\operatorname{Inn} \mathbb{A}=\operatorname{Inn}_{\mathbb{A}} \mathbb{A}$. 
Lemma 5.1. Let $\mathbb{A}$ be a Leibniz algebra, and $A$ its ideal. Then the followings hold:

(i) If $A$ is a $\Delta$-ideal of $\mathbb{A}$, then $\operatorname{Inn}_{A} \mathbb{A}$ is an ideal of $D$,

(ii) $\operatorname{Inn}_{A} \mathbb{A}$ is an ideal of $\operatorname{Inn} \mathbb{A}$,

(iii) $\operatorname{Inn}_{A} \mathbb{A}=0$ iff $A \subseteq \operatorname{rann} \mathbb{A}$,

(iv) $\operatorname{Inn} \mathbb{A}=0$ iff $\mathbb{A}^{2}=0$,

(v) $\operatorname{Inn}_{A} \mathbb{A}=\operatorname{Inn} \mathbb{A}$ iff $\mathbb{A}=A+\operatorname{rann} \mathbb{A}$,

(vi) $\operatorname{rann} \mathbb{A}$ is a $D$-ideal of $\mathbb{A}$,

(vii) There is the Lie algebra isomorphism

$$
\operatorname{Inn} \mathbb{A} \ni r_{a} \mapsto a+\operatorname{rann} \mathbb{A} \in \mathbb{A} / \operatorname{rann} \mathbb{A}
$$

(viii) If $\Phi$ is an ideal of $\operatorname{Inn} \mathbb{A}$, then

$$
\Delta_{\Phi}=\left\{a \in \mathbb{A} \mid r_{a} \in \Phi\right\}
$$

is an ideal of $\mathbb{A}$,

(ix) If $\Phi$ is an ideal of $D$, then $\Delta_{\Phi}$ is a D-ideal of $\mathbb{A}$,

(x) If $B, C \subseteq \mathbb{A}$, then $\left[\operatorname{Inn}_{B} \mathbb{A}, \operatorname{Inn}_{C} \mathbb{A}\right]=\operatorname{Inn}_{C B} \mathbb{A}$.

Proof. This follows from routine calculations.

Lemma 5.2. Let $A$ be a Leibniz algebra and $\Phi$ an ideal of $D$. Then we have:

(i) ADer $\mathbb{A}$ is an ideal of $D$,

(ii) $[\Phi, \operatorname{Inn} \mathbb{A}]=0$ iff $\Phi \subseteq \operatorname{ADer} \mathbb{A}$,

(iii) If $\Phi \cap \operatorname{Inn} \mathbb{A}=0$, then $\Phi \subseteq \mathrm{ADer} \mathbb{A}$.

Corollary 5.1. Let $\mathbb{A}$ be a Leibniz algebra. Then $\operatorname{Inn} \mathbb{A}$ is a simple (respectively prime, semisimple or primary) Lie algebra if and only if $\mathbb{A}$ is a simple (respectively prime, semisimple or primary) Leibniz algebra.

Proof. (i) Follows immediately.

(ii) Let $\delta \in \Phi$ and $a \in \mathbb{A}$.

$(\Leftarrow)$ Since

$$
r_{\delta(a)}=\left[\delta, r_{a}\right]
$$

and $\left[\delta, r_{a}\right]=0$, we deduce that $\delta(a) \in \operatorname{rann} \mathbb{A}$.

$(\Rightarrow)$ Inasmuch $r_{\delta(a)}=0$, we conclude that the assertion holds in view of Eq. (5.15).

(iii) This follows from (ii).

A proof of Proposition $\mathbf{5 . 1}$ is based on Lemmas above and is omitted. 


\subsection{Generalized derivations}

A linear mapping $T: \mathbb{A} \rightarrow \mathbb{A}$ is called a multiplier of a Leibniz algebra $\mathbb{A}$ if

$$
T(x y)=T(x) y
$$

for all $x, y \in \mathbb{A}$. The set of all multipliers of $\mathbb{A}$ is denoted by $\mathrm{M}(\mathbb{A})$. Obviously that, for any $T \in \mathrm{M}(\mathbb{A})$, $(T, 0) \in \operatorname{IGDer} \mathbb{A}$ and so

$$
\mathrm{M}(\mathbb{A}) \subseteq \operatorname{IGDer} \mathbb{A} \subseteq \text { GDer } \mathbb{A}
$$

Below we will formulate some important statements concerning the generalized derivations and their applications to studying the structure of the Leibniz algebra. There holds the following lemma.

Lemma 5.3. Let $\mathbb{A}$ be a Leibniz algebra. Then:

(i) GDer $\mathbb{A}$ is a Lie ring with respect to the point-wise addition "+" and the point-wise Lie multiplication "[-, $]$ " given by the rules

$$
(H+K)(x)=H(x)+K(x)
$$

and

$$
[H, K](x)=H(K(x))-K(H(x))
$$

for all $x \in \mathbb{A}$ and $H, K \in$ GDer $\mathbb{A}$,

(ii) if $A$ is a $D$-ideal of $\mathbb{A}$, then

$$
\mathrm{I}_{\mathrm{A}} \text { GDer } \mathbb{A}=\left\{F \in \mathrm{GDer} \mathbb{A} \mid F \text { is associated with some } r_{a} \in \operatorname{Inn}_{A} \mathbb{A}\right\}
$$

is an ideal of GDer $\mathbb{A}$ (in particular, IGDer $\mathbb{A}=\mathrm{I}_{\mathbb{A}} \operatorname{GDer} \mathbb{A}$ and $\mathrm{M}(\mathbb{A})=\mathrm{I}_{0} \mathrm{GDer} \mathbb{A}$ ),

(iii) $\operatorname{GDer} \mathbb{A}=\mathrm{M}(\mathbb{A})+\operatorname{Der} \mathbb{A}$, where $\mathrm{M}(\mathbb{A})$ is an ideal of $\mathrm{GDer} \mathbb{A}$, and

$$
\mathrm{M}(\mathbb{A}) \bigcap \operatorname{Der} \mathbb{A} \subseteq \operatorname{ADer} \mathbb{A},
$$

(iv) $\operatorname{IGDer} \mathbb{A}=\mathrm{M}(\mathbb{A})+\operatorname{Inn} \mathbb{A}$, where $\mathrm{M}(\mathbb{A})$ is an ideal of $\operatorname{IGDer} \mathbb{A}$, and

$$
\mathrm{M}(\mathbb{A}) \bigcap \operatorname{Inn} \mathbb{A} \subseteq \operatorname{ADer} \mathbb{A},
$$

(v) if $(F, \delta),(F, d) \in \operatorname{GDer} \mathbb{A}$, then $\delta+\operatorname{ADer} \mathbb{A}=d+\operatorname{ADer} \mathbb{A}$,

(vi) if $\left(F, r_{a}\right),\left(F, r_{b}\right) \in \operatorname{IGDer} \mathbb{A}$ (where $\left.a, b \in \mathbb{A}\right)$, then $\mathbb{A}(a-b) \subseteq \operatorname{rann} \mathbb{A}$.

Its proof is standard and is omitted. Here we need to remark that Lie algebras $\mathbb{A}$ with abelian derivation algebras Der $\mathbb{A}$ were studied by S. Tôgô [79]. In particular, the next lemmas hold.

Lemma 5.4. Let $\mathbb{A}$ be a Leibniz algebra and $(F, d) \in \operatorname{GDer} \mathbb{A}$. Then we have:

(i) If $F=0$, then $d \in \mathrm{ADer} \mathbb{A}$,

(ii) If $d \in \mathrm{ADer} \mathbb{A}$, then $F \in \mathrm{M}(\mathbb{A})$,

(iii) If GDer $\mathbb{A}$ is an abelian Lie algebra, then Der $\mathbb{A}$ is abelian,

(iv) If $\mathbb{A} \neq 0$, then $\operatorname{IGDer} \mathbb{A} \neq 0$. 
Proof. Assume that $x, y \in \mathbb{A}$.

(i) In fact,

$$
0=F(x y)=F(x) y+x d(y)=x d(y)
$$

and $\operatorname{so} d(y) \in \operatorname{rann} \mathbb{A}$.

(ii) Since $F(x y)=F(x) y$, we deduce that $F \in \mathrm{M}(\mathbb{A})$.

(iii) We have Der $\mathbb{A} \subseteq$ GDer $\mathbb{A}$ and therefore the assertion holds.

(iv) This can be verified in a straightforward manner.

Lemma 5.5. Let $\mathbb{A}$ be a Leibniz algebra and $\left(H, r_{a}\right) \in \mathrm{IGDer} \mathbb{A}$. Then the following hold:

(i) If $H=0$, then $a \in \operatorname{rann} \mathbb{A}$,

(ii) If $a \in \operatorname{rann} \mathbb{A}$, then $H \in \mathrm{M}(\mathbb{A})$,

(iii) If IGDer $\mathbb{A}$ is an abelian Lie algebra, then $\mathbb{A}^{2} \subseteq \operatorname{rann} \mathbb{A}$,

(iv) If $\mathbb{A}$ is abelian, then $\operatorname{IGDer} \mathbb{A}=\mathrm{M}(\mathbb{A})$.

Proof. The properties $(i)-(i v)$ are readily verified using the same argument as in the proof of Lemma 5.4 .

Let $\Phi \subseteq \operatorname{GDer} \mathbb{A}, \Gamma \subseteq \operatorname{Der} \mathbb{A}$,

$$
\begin{gathered}
T_{\Phi}=\{d \in \operatorname{Der} \mathbb{A} \mid \text { there is } H \in \Phi \text { that is associated with } d \in \operatorname{Der} L\}, \\
U_{\Gamma}=\{H \in \operatorname{GDer} \mathbb{A} \mid H \text { is associated with some } d \in \Gamma\}
\end{gathered}
$$

and

$$
\Sigma_{\Phi}=\left\{a \in \mathbb{A} \mid \text { there is } H \in \Phi \text { that is associated with } r_{a} \in \operatorname{Inn} \mathbb{A}\right\} .
$$

Lemma 5.6. Let $\mathbb{A}$ be a Leibniz algebra. If $\Phi$ is an ideal of GDer $\mathbb{A}$ such that $[\Phi$, IGDer $\mathbb{A}]=0$, then $T_{\Phi} \subseteq \mathrm{ADer} \mathbb{A}$ (and so $\Phi \subseteq \mathrm{M}(\mathbb{A})$ ).

Proof. Indeed, if $(F, d) \in \Phi$, then $r_{d(a)}=\left[d, r_{a}\right] \in \operatorname{ADer} \mathbb{A}$ for any $a \in \mathbb{A}$ and so $d(a) \in \operatorname{rann} \mathbb{A}$.

Lemma 5.7. Let $\mathbb{A}$ be a Leibniz algebra. Then the following hold:

(1) If $\Phi$ is an ideal of IGDer $\mathbb{A}$ (respectively GDer $\mathbb{A}$ ), then $\Sigma_{\Phi}$ is an ideal (respectively a $D$ ideal) of $\mathbb{A}$,

(2) If $\Gamma$ is an ideal of Der $\mathbb{A}$, then $U_{\Gamma}$ is an ideal of GDer $\mathbb{A}$ (in particular, $U_{0}=\mathrm{I}_{0}$ GDer $\mathbb{A}=$ $\mathrm{M}(\mathbb{A}))$

(3) If $\Phi$ is an ideal of GDer $\mathbb{A}$, then $T_{\Phi}$ is an ideal of $\operatorname{Der} \mathbb{A}$.

Proof. (1) Let $a, b \in \Sigma_{\Phi}, t \in \mathbb{A},\left(H, r_{a}\right),\left(K, r_{b}\right) \in \Phi,\left(S, r_{t}\right) \in \operatorname{IGDer} \mathbb{A}$ and $(M, \delta) \in$ GDer $\mathbb{A}$. Since

$$
\left(H-K, r_{a-b}\right),\left([M, H], r_{\delta(a)}\right),\left([H, S], r_{t a}\right),\left([S, H], r_{a t}\right) \in \Phi,
$$

we conclude that $a-b, \delta(a), t a, a t \in \Sigma_{\Phi}$ and therefore $\Sigma_{\Phi}$ is an ideal of $\mathbb{A}$.

Then (2)-(3) can be proved by the same argument as in the part (1).

Lemma 5.8. Let $\mathbb{A}$ be a Leibniz algebra and $A$ its ideal. Then the following conditions are equivalent: 
(1) $\mathrm{I}_{\mathrm{A}} \mathrm{GDer} \mathbb{A} \subseteq \mathrm{M}(\mathbb{A})$,

(2) $A \subseteq \operatorname{rann} \mathbb{A}$,

(3) $\operatorname{Inn}_{A} \mathbb{A}=0$.

Proof. For a proof, see Lemmas 5.1 and 5.3.

Lemma 5.9. Let $\mathbb{A}$ be a Leibniz algebra and $a \in \mathbb{A}$. If $\mathbb{A}$ is $D$-semisimple, then $r_{a} \in \mathrm{ADer} \mathbb{A}$ iff $a \in \operatorname{rann} \mathbb{A}$.

Proof. We have that

$$
\mathbb{A} a=r_{a}(\mathbb{A}) \subseteq \operatorname{rann} \mathbb{A} .
$$

Moreover, $\mathbb{A} \delta^{n}(a) \subseteq$ rann $\mathbb{A}$ for any $\delta \in D$ and a non-negative integer $n$. If $l, t \in \mathbb{A}$, then

$$
0=t\left(l \delta^{n}(a)\right)=(t l) \delta^{n}(a)-\left(t \delta^{n}(a)\right) l
$$

and so $\left(t \delta^{n}(a)\right) l \in \mathbb{A} \delta^{n}(a)$. Hence

$$
\mathbb{A}_{0}:=\mathbb{A} a+\sum_{n=1}^{\infty} \mathbb{A} \delta^{n}(a)
$$

is a $D$-ideal of $\mathbb{A}$ and $\mathbb{A}_{0} \cdot \mathbb{A}_{0}=0$. Consequently $\mathbb{A} a=0$.

Lemma 5.10. Let $\mathbb{A}$ be a Leibniz algebra and $A$ its ideal. Then there exist Lie algebra isomorphisms:

(1)

$$
\operatorname{Der} \mathbb{A} / \operatorname{ADer} \mathbb{A} \ni d+\operatorname{ADer} \mathbb{A} \mapsto F+\mathrm{M}(\mathbb{A}) \in \operatorname{GDer} \mathbb{A} / \mathrm{M}(\mathbb{A}),
$$

(2)

where $(F, d) \in \operatorname{GDer} \mathbb{A}$,

$$
\operatorname{Inn} \mathbb{A} / \operatorname{AInn} \mathbb{A} \ni r_{a}+\operatorname{AInn} \mathbb{A} \mapsto H+\mathrm{M}(\mathbb{A}) \in \operatorname{IGDer} \mathbb{A} / \mathrm{M}(\mathbb{A}),
$$

where $\left(H, r_{a}\right) \in \operatorname{IGDer} \mathbb{A}$.

Proof. It is straightforward to verify the above properties.

Based now on Lemmas 5.8, 5.9 and 5.10 one proved Theorem 5.1.

Proof. (a) Let $\mathbb{A}$ be a $D$-prime Leibniz algebra and $\Psi, \Omega$ be ideals of $D$ such that $[\Psi, \Omega]=0$. Then $\operatorname{rann} \mathbb{A}=\operatorname{Leib}(\mathbb{A})$. If

$$
\Phi:=\Psi \bigcap \operatorname{Inn} \mathbb{A} \text { and } \Lambda:=\Omega \bigcap \operatorname{Inn} \mathbb{A},
$$

then

$$
\Phi=\operatorname{Inn}_{\Delta_{\Phi}} \mathbb{A}, \Lambda=\operatorname{Inn}_{\Delta_{\Lambda}} \mathbb{A} \text { and }[\Phi, \Lambda]=0 .
$$

Lemma 5.1(x) and (iii) imply that

$$
\Delta_{\Lambda} \Delta_{\Phi} \subseteq \operatorname{rann} \mathbb{A} .
$$


Since $\Delta_{\Lambda}$ and $\Delta_{\Phi}$ are $D$-ideals by Lemma 5.1(ix), we deduce that

$$
\Delta_{\Lambda} \subseteq \operatorname{rann} \mathbb{A} \text { or } \Delta_{\Phi} \subseteq \operatorname{rann} \mathbb{A}
$$

by the $D$-primeness of $\mathbb{A}$. This gives that $\Lambda=0$ or $\Phi=0$ by Lemma 5.1(iii). As a consequence of Lemma 5.2(iii), the quotient Lie algebra $D /$ ADer $\mathbb{A}$ is prime.

(b) If $\mathbb{A}$ is a $D$-semisimple Leibniz algebra, then we can deduce that $D / A D e r \mathbb{A}$ is semisimple as in $(a)$.

\subsection{Case of the finite inner derivation algebra}

Lemma 5.11. Let $\mathbb{A}$ be a Leibniz ring (in particular, a Leibniz algebra) and $a \in \mathbb{A}$. Then the following hold:

(1) the left centralizer $C_{l, \mathbb{A}}(a):=\{u \in \mathbb{A} \mid u a=0\}$ of $a$ is a subring (respectively a subalgebra) of $\mathbb{A}$ and lann $\mathbb{A} \subseteq C_{l, \mathbb{A}}(a)$,

(2) the right centralizer $C_{r, \mathbb{A}}(a):=\{u \in \mathbb{A} \mid a u=0\}$ of a is a subring (respectively a subalgebra) of $\mathbb{A}$ and $\operatorname{rann} \mathbb{A} \subseteq C_{r, \mathbb{A}}(a)$.

Proof. (1) In fact, if $u, v \in C_{l, \mathbb{A}}(a)$, then $u a=0=v a$ and so $(u v) a=u(v a)+(u a) v=0$.

(2) By the same argument as (1).

By analogy with J. Lewin [50, Lemma 1], we obtain the next

Proposition 5.2. Let $\mathbb{A}$ be a Leibniz ring (in particular, a Leibniz algebra). If $S$ is a subring (respectively a subalgebra) of finite index in $\mathbb{A}$, then $\mathbb{A}$ has an ideal I of finite index such that $I \subseteq S$.

Proof. Suppose that $|\mathbb{A}: S|=m$ for some positive integer $m$ and $T:=\left\{a_{1}, \ldots, a_{m}\right\}$ be a transversal for the subgroup $S$ of the additive group $(\mathbb{A},+)$. Let $s \in S, n \geq 2$ be an integer and $W_{n}$ be the set of all words $w(x, t)$ of length $n$ over the set $T$ that contains two indeterminate $x$ and $t$ every only one time and such that $w(a, s) \in \mathbb{A}$ for any $a \in \mathbb{A}, s \in S$

$$
\varphi_{w, s}: \mathbb{A} / S \ni a+S \mapsto w(a, s)+S \in \mathbb{A} / S
$$

is an endomorphism of the additive group $\mathbb{A} / S$. The length of $w(x, t)$ is the number of all its symbols from $T \cup\{x, t\}$ and the word $w(x, t)$ of length 2 is of the form $x t$ or $t x$. Since $\mathbb{A} / S$ is finite, its endomorphism $\operatorname{ring} \operatorname{End}(\mathbb{A} / S)$ is finite too. Then

$$
\varphi_{w}: S \ni s \mapsto \varphi_{w, s} \in \operatorname{End}(\mathbb{A} / S)
$$

is a group homomorphism and its kernel $K_{w}:=\operatorname{Ker} \varphi_{w}$ has a finite index in $S$. The set $W_{n}$ is finite and so

$$
I_{n}:=\bigcap_{w \in W_{n}} K_{w}
$$

has a finite index in $S$. Moreover, $I_{n} \subseteq S$,

$$
I:=\sum_{n=2}^{\infty} I_{n}
$$

is an ideal of $\mathbb{A}$ and $|\mathbb{A}: I|<\infty$. 
Corollary 5.2. Let $\mathbb{A}$ be a Leibniz ring (in particular, a Leibniz algebra) and $a \in \mathbb{A}$. Then the following hold:

(1) if $\left|\mathbb{A}: C_{l, \mathbb{A}}(a)\right|<\infty$, then there exists an ideal $L_{a}$ of $\mathbb{A}$ such that $L_{a} a=0$ and $a L_{a} \subseteq \operatorname{Leib}(\mathbb{A})$,

(2) if $\left|\mathbb{A}: C_{r, \mathbb{A}}(a)\right|<\infty$, then there exists an ideal $R_{a}$ of $\mathbb{A}$ such that $a R_{a}=0$ and $R_{a} a \subseteq \operatorname{Leib}(\mathbb{A})$.

Proof. It is straightforward to verify the above properties.

Remark 5.3. A Leibniz algebra $\mathbb{A}$ over an infinite field does not contain a proper subalgebra of finite index.

Proof of Theorem 5.2. (1) $\Rightarrow$ (2) Since the additive quotient group

$$
\mathbb{A} / \operatorname{rann} \mathbb{A}=\left\{a_{1}+\operatorname{rann} \mathbb{A}, \ldots, a_{m}+\operatorname{rann} \mathbb{A}\right\}
$$

for some positive integer $m$ and $a_{1}, \ldots, a_{m} \in \mathbb{A}$ and, for every $x \in \mathbb{A}$, there exists an integer $i=i(x)$ $(1 \leq i \leq m)$ such that $x \in a_{i}+\operatorname{rann} \mathbb{A}$, we see that $r_{x}=r_{a_{i}}$. Then

$$
\operatorname{Inn} \mathbb{A}=\left\{r_{x} \mid x \in \mathbb{A}\right\}=\left\{r_{a_{i}} \mid i=1, \ldots, m\right\}
$$

is finite.

$(2) \Rightarrow(1)$ Suppose that Inn $\mathbb{A}=\left\{r_{u_{i}} \mid i=1, \ldots, m\right\}$ for some positive integer $m$ and $u_{1}, \ldots, u_{m} \in$ $\mathbb{A}$. Then, for any $x \in \mathbb{A}, r_{x} \in \operatorname{Inn} \mathbb{A}$ and so $r_{x}=r_{u_{j}}$ for some integer $j=j(x)(1 \leq j \leq m)$. Then $\mathbb{A}\left(x-u_{i}\right)=0$ and therefore $x \in u_{i}+\operatorname{rann} \mathbb{A}$. This means that

$$
\mathbb{A} / \operatorname{rann} \mathbb{A}=\left\{u_{i}+\operatorname{rann} \mathbb{A} \mid i=1, \ldots, m\right\}
$$

is finite.

Lemma 5.12. Let $\mathbb{A}$ be a Leibniz ring (in particular, a Leibniz algebra). Then the following hold:

(1) if $|\mathbb{A}: Z(\mathbb{A})|<\infty$, then the commutator ideal $\mathbb{A}^{(2)}$ is finitely generated (in particular, if $\mathbb{A}$ is algebra, then $\mathbb{A}^{2}$ is finite),

(2) if $\mathbb{A}^{(2)}$ is finite, then $\left|\mathbb{A}: C_{l, \mathbb{A}}(a)\right|<\infty$ for any $a \in \mathbb{A}$.

Proof. (1) Let $\left\{b_{1}, \ldots, b_{s}\right\}$ be a transversal for the subgroup $Z(\mathbb{A})$ of the additive group $(\mathbb{A},+)$. Then, for any $x, y \in \mathbb{A}$, there exist integers $i=i(x)$ and $j=j(y)$ such that $x \in b_{i}+Z(\mathbb{A})$ and $y \in$ $b_{j}+Z(\mathbb{A})$. Then $x y=b_{i} b_{j}$ and so $\mathbb{A}^{(2)}$ is finitely generated.

(2) Assume that $a \in \mathbb{A}$. Then the kernel $\operatorname{Ker}\left(r_{a}\right):=\{u \in \mathbb{A} \mid u a=0\}=C_{l, \mathbb{A}}(a)$ and there exists a group isomorphism

$$
\mathbb{A} / \operatorname{Ker}\left(r_{a}\right) \cong \mathbb{A} a
$$

Since $\mathbb{A} a \subseteq \mathbb{A}^{(2)}$, the assertion holds.

\section{Supplement: The classical Poisson manifolds approach revisited}

It is interesting to look at the construction of the Hamiltonian operators presented above and revisit it from the standard point of view, considering them as those defined on the naturally associated 
$[1,4,18,20,60,61,69]$ cotangent space $T^{*}(M)$ to some linear functional manifold $M \simeq \widetilde{\mathbb{A}}$. Then, a Hamiltonian operator on $M$ is defined [1] as a smooth mapping $\vartheta: M \rightarrow \operatorname{Hom}\left(T^{*}(M) ; T(M)\right)$, such that for any fixed $u \in M$ the bracket

$$
\{f, g\}:=(\nabla f(u), \vartheta(u) \nabla g(u))
$$

where $f, g: M \rightarrow \mathbb{K}$ are arbitrary smooth mappings from the functional space $\mathscr{D}(M) \simeq \mathscr{F}_{\widetilde{\mathbb{A}}}(u)$, satisfies the Jacobi identity. The bracket (6.1) is determined on $M$ by means of the natural convolution $(\cdot, \cdot)$ on the product $T^{*}(M) \times T(M)$, and respectively, the gradient $\nabla f(u) \in T^{*}(M)$ of a function $f \in \mathscr{D}(M)$ is calculated as

$$
(\nabla f(u), h):=d f[u+\varepsilon h] /\left.d \varepsilon\right|_{\varepsilon=0}
$$

for any $h \in T(M)$. It is well known [41,52] that a linear operator $\vartheta(u): T^{*}(M) \rightarrow T(M)$, determined at any point $u \in M$, is Hamiltonian iff the suitably defined [41] Schouten-Nijenhuis bracket

$$
[[\vartheta(u), \vartheta(u)]]=0
$$

identically on $M$. Namely, this condition (6.3) was used in the investigations [41,77] to formulate criteria for the operator $\vartheta(u): T^{*}(M) \rightarrow T(M)$ to be Hamiltonian on the functional manifold $M$. Yet these criteria appear to be very complicated and involve a large amount of cumbersome calculations even in the case of fairly simple differential expressions. So, we have reanalyzed this problem from a slightly different point of view. First, recall that the Jacobi identity for the bracket (6.1) is completely equivalent to the fact that the bracket operator defined as $D_{f}(g):=\{f, g\}$ for a fixed $f \in \mathscr{D}(M)$ and arbitrary $g \in \mathscr{D}(M)$ acts as a differentiation on the space $(\mathscr{D}(M) ;\{\cdot, \cdot\})$ :

$$
D_{f}\{g, h\}=\left\{D_{f}(g), h\right\}+\left\{g, D_{f}(h)\right\}
$$

where $g, h \in \mathscr{D}(M)$ are taken arbitrary. This can be easily reformulated as follows: take any element $\varphi \in T^{*}(M)$, such that the Fréchet derivative $\varphi^{\prime}(u)=\varphi^{\prime, *}(u)$ at any $u \in M$ with respect to the convolution $(\cdot, \cdot)$ on $T^{*}(M) \times T(M)$, and construct a vector field $K: M \rightarrow T(M)$ as

$$
K(u):=\vartheta(u) \varphi(u)
$$

Then the differentiation condition (6.4) can be equivalently rewritten $[1,18,60,61,69]$ as the strong Lie derivative

$$
L_{K} \vartheta:=\vartheta^{\prime} \cdot K-\vartheta K^{\prime, *}-K^{\prime} \vartheta=0
$$

along the vector field $K(u)=\vartheta(u) \varphi(u) \in T(M)$ at any $u \in M$ for all "self-adjoint" elements $\varphi \in T^{*}(M)$. Equivalently, a given linear skew-symmetric operator $\vartheta(u): T^{*}(M) \rightarrow T(M), u \in M$, is Hamiltonian iff the Lie derivative (6.6) vanishes for all "self-adjoint" elements $\varphi \in T^{*}(M)$. Moreover, as was observed in [56], it suffices to check the condition (6.6) only on the subspace of elements $\varphi \in T^{*}(M)$ satisfying the condition $\varphi^{\prime}(u)=0$ for any $u \in M$. 
As an example, one can check that a skew-symmetric matrix-differential operator on $M$ of the form

$$
\vartheta(u):=\sigma(u) D_{x}+D_{x} \sigma^{\top}(u)
$$

where, an $n$-dimensional square matrix $\sigma(u):=\left(\sum_{s=1}^{n} u_{s} \sigma_{i j}^{s}: i, j=\overline{1, n}, n \in \mathbb{Z}_{+}\right), u \in M$, satisfies the condition (6.6) iff the linearly independent elements from $\operatorname{span}_{\mathbb{K}}\left\{e_{j} \in \mathbb{A}: j=\overline{1, n}\right\}$ generate the finite dimensional nonassociative Novikov algebra (5.4) and satisfy the conditions $e_{i} \circ e_{j}=\sum_{s=1}^{n} \sigma_{i j}^{s} e_{s}$ for all $i, j=\overline{1, n}$. Similarly, one can verify that the skew-symmetric inverse-differential operator

$$
\vartheta(u):=\sigma(u) D_{x}^{-1}+D_{x}^{-1} \sigma(u)^{\top},
$$

where, as above $\sigma(u):=\left(\sum_{s=1}^{n} u_{s} \sigma_{i j}^{s}: u \in M, i, j=\overline{1, n}, n \in \mathbb{Z}_{+}\right)$, the sign " $\mathrm{T}$ " means the usual matrix transposition, is Hamiltonian iff the basic non-associative algebra $\mathbb{A}:=\underset{\mathbb{K}}{\operatorname{span}}\left\{e_{j}: j=\overline{1, n}\right\}$ coincides with the right Leibniz algebra (5.7) and the condition $e_{i} \circ e_{j}=\sum_{s=1}^{n} \sigma_{i j}^{s} e_{s}$ holds for any $i, j=\overline{1, n}$. The skew-symmetric inverse-differential operator (6.8) can be naturally generalized to the expression

$$
\vartheta(u):=D_{x} \sigma(u) D_{x}^{-1}-D_{x}^{-1} \sigma(u)^{\top} D_{x},
$$

which can be rewritten as

$$
\vartheta(u)=\sigma\left(D_{x} u\right) D_{x}^{-1}+D_{x}^{-1} \sigma\left(D_{x} u\right)^{\top}+\sigma(u)-\sigma(u)^{\top} .
$$

The condition (6.6) for the operator (6.10) to be Hamiltonian reduces to the constraints on the related nonassociative algebra $\mathbb{A}:=\underset{\mathbb{K}}{\operatorname{span}}\left\{e_{j}: j=\overline{1, n}\right\}$ exactly coinciding with that of (5.9), and analyzed in some detail in Section 3.

As was already mentioned, based on the matrix representations of the right Leibniz algebra (5.7) and the new non-associative Riemann algebra (5.10) one can construct Hamiltonian operators $\vartheta(u): \mathscr{L}_{\widetilde{\mathbb{A}}} \rightarrow \mathscr{L}_{\widetilde{\mathbb{A}}}$ on the associated weak Lie algebra $\mathscr{L}_{\widetilde{\mathbb{A}}}$ suitable for describing a wide class of multicomponent hierarchies $[19,67,68]$ of integrable Riemann hydrodynamic systems and their various reductions.

\section{Conclusions}

In this work we succeeded in devising a tensor and differential-algebraic reformulation of the criteria $[41,56,77]$ for a given differential expression to be Hamiltonian and developed an effective approach to classification of the algebraic Poisson structures underlying the integrable multicomponent Hamiltonian systems. We have created a simple algorithm allowing to construct new algebraic structures within which the corresponding Hamiltonian operators exist and generate integrable multicomponent dynamical systems. We also showed, as examples, that the well-known Novikov algebraic structure, obtained in $[12,41]$ as a condition for a matrix differential expression to be Hamiltonian, is in the devised approach a differentiation on the weak adjacent Lie algebra, naturally associated with a suitably constructed differential loop algebra. The related algebraic structures of the right Leibniz algebra important for its representations, are studied in some details. It is mentioned 
that constructed Hamiltonian operators describe a wide class of multicomponent hierarchies $[19,67]$ of integrable multicomponent Riemann hydrodynamic Riemann systems. Their reductions appear to be closely related both to the integrable Camassa-Holm and with the Degasperis-Processi dynamical systems, and are of special interest from the equivalence transformation point of view devised recently in [80]. A brief revisit to the classical Poisson manifold method of constructing Hamiltonian operators on functional manifolds has also been included to contrast it with our new approach.

\section{Acknowledgements}

The authors cordially thank Prof. Maciej Błaszak (Poznań University, Poland), Jan Cieśliński (Białystok University, Poland) and Prof. Maxim Pavlov (Novosibirsk State University) for their cooperation and useful discussions of the results obtained. A.P. is cordially indebted to Prof. V.E. Zakharov (University of Arizona, Tucson) and Prof. J. Szmigelski (University of Saskatchewan, Saskatoon) for their interest in the work and instructive discussions during the XXXV Workshop on Geometric Methods in Physics, held 26.06-2.07.2016 in Białowieża, Poland.

\section{References}

[1] R. Abraham, J.E. Marsden. Foundations of Mechanics. Benjamin/Cummings Publisher, 1978.

[2] S. Albeverio, Sh.A. Ayupov and B.A. Omirov, On nilpotent and simple Leibniz algebras, Comm. Algebra 33(2005) 159-172.

[3] M. Amini, I. Rakhimov, S.J. Langari, Enveloping Lie Algebras of Low Dimensional Leibniz Algebras. Applied Mathematics 2(2011) 1027-1030.

[4] V.I. Arnold, Mathematical Methods of Classical Mechanics. Springer (1989).

[5] S. Ayupov, B. Omirov, On Leibniz Algebras, In: Algebra and Operator Theory (Tashkent, 1997), Kluwer Acad. Publ., Dordrecht, 1998, pp. 1-12.

[6] C. Bai and D. Meng, Addendum: invariant bilinear forms, J. Phys. A 34(2001) 8193-8197.

[7] C. Bai and D. Meng, Transitive Novikov algebras on four-dimensional nilpotent Lie algebras, Int. J. Theor. Phys. 40(2001) 1761-1768.

[8] C. Bai and D. Meng, The classification of Novikov algebras in low dimensions, J. Phys. A 34(2001) 1581-1594.

[9] I. Bajo, Lie algebras admitting non-singular prederivations, Indag. Math. 8(1997) 433-437.

[10] A.A. Balinsky, A.I. Balinsky, On the algebraic structures connected with the linear Poisson brackets of hydrodynamics type. arXiv:hep-th/9311134v1 22; J. Phys. A: Math. Gen. 26(1993) L361.

[11] A.A. Balinsky, Yu.M. Burman. Quadratic Poisson brackets compatible with an algebra structure. arXiv:hep-th/9407041v2 10 Jul 1994.

[12] A.A. Balinski, S.P. Novikov, Poisson brackets of hydrodynamic type, Frobenius algebras and Lie algebras, Sov. Math. Dokl. 32(1985) 228-231.

[13] A.A. Balinsky, Yu.M. Burman, Quadratic Poisson brackets and Drinfel'd theory for associative algebras. arXiv:q-alg/9501019v1 16 Jan 1995.

[14] D.W. Barnes, Faithful representation of Leibniz algebras, Proc. Amer. Math. Soc. 141(2013) 29912995.

[15] A. Belavin and V. Drinfel'd, Solutions of the classical Yang-Baxter equation for simple Lie algebras (Russian), Funktsional. Anal. i Prilozhen. (3) 16 (1982) 1-29; english traslated in Functional Anal. Appl. (3) 16 (1982) 159-180 (1983).

[16] A. Belavin and V. Drinfel'd, The classical Yang-Baxter equation for simple Lie algebras (Russian), Funktsional, Anal. i Prilozhen. (3) 17 (1983) 69-70; english traslated in Functional Anal. Appl. (3) 17(1983) 220-221.

[17] Y. Benoist, Une nilvariete non-affine, J. Diff. Geom. 41(1995) 21-52.

[18] D. Blackmore, A.K. Prykarpatsky and V.H. Samoylenko. Nonlinear Dynamical Systems of Mathematical Physics. World Scientific Publisher, NJ, USA, 2011. 
[19] D. Blackmore, Ya.A. Prykarpatsky, N.N. Bogolubov (Jr.) and A.K. Prykarpatski, Integrability of and differential-algebraic structures for spatially 1D hydrodynamical systems of Riemann type. Chaos, Solitons \& Fractals 59(2014) 59-81.

[20] M. Błaszak, Bi-Hamiltonian Dynamical Systems. NY, Springer, 1998.

[21] A.M. Bloh, On a generalization of the concept of Lie algebra, Dokl. Akad. Nauk SSSR 165(1965), 471-473.

[22] A.M. Bloh, Cartan-Eilenberg homology theory for a generalized class of Lie algebras, Dokl. Akad. Nauk SSSR 175(1967), 824-826.

[23] M. Brešar, On the distance of the composition of two derivations to the generalized derivations, Glasgow J. Math. 33(1991) 89-93.

[24] M. Brešar, Introduction to Noncommutative Algebra. NY, Springer, 2014.

[25] D. Burde, Affine structures on nilmanifolds, Int. J. Math. 7(1996) 599-616.

[26] D. Burde and W. de Graaf, Classification of Novikov algebras, Applicable Algebra in Engineering, Communication and Computing, (1) 24(2013) 1-15.

[27] D. Burde, W.A. Moens, Periodic derivations and prederivations of Lie algebras, J. Algebra 357(2012) 208-221.

[28] R. Camassa and D.D. Holm, An integrable shallow water wave equation with peaked solitons, Phys. Rev. Lett. 71(1993) 1661-1664. .

[29] M. Chen M, S.-Q. Liu and Y. Zhang, A two-component generalization of the Camassa-Holm equation and its solutions, Lett. Math. Phys. 75(2006) 1-15.

[30] A. Degasperis, D.D. Holm and A.N.W. Hone, A new integrable equation with peakon solutions (Russian), Theoret. Math. Fiz. (2) 133(2002) 170-183; english traslated in Theoret. and Math. Phys. (2) 133(2002) 1463-1474.

[31] A. Degasperis, M. Procesi, Asymptotic integrability. In: Degasperis A, Gaeta G, editors. Symmetry and Perturbation Theory. Singapore: World Scientific, 1999.

[32] I. Demir, K.C. Misra, E. Stitzinger, On some structures of Leibniz algebras, In: Recent Advances in Representation Theory, Quantum Groups, Algebraic Geometry, and Related Topics, Contemporary Math. 623, Amer. Math. Soc., Providence, RI, 2014, pp. 41-54.

[33] I. Dorfman, Dirac Structures and Integrability of Nonlinear Evolution Equations, Wiley, Chichester, U.K., 1993.

[34] B.A. Dubrovin and S.P. Novikov, Hamiltonian formalism of one-dimensional systems of hydrodynamic type and the Bogolyubov-Whitham averaging method, Sov. Math. Dokl. 27(1983) 665-669.

[35] B.A. Dubrovin and S.P. Novikov, On Poisson brackets of hydrodynamic type, Sov. Math. Dokl. 30(1984) 651-654.

[36] L.D. Faddeev, L.A. Takhtadjan, Hamiltonian Methods in the Theory of Solitons. Springer, New York, Berlin, 1986.

[37] G. Falqui. On a Camassa-Holm type equation with two dependent variables, J. Phys. A: Math. Gen. 39(2006) 327-342.

[38] R. Felipe, N. Lorpez-Reyes and F.R. Onga, Matrices for Leibniz Algebras. Letters in Math. Phys. 63(2003) 157-164.

[39] A. Fialowski, A.Kh. Khudoyberdiyev, B.A. Omirov, A characterization of nilpotent Leibniz algebras, Algebr. Represent. Theory 16(2013) 1489-1505.

[40] A. Fialowski, É.Zs. Mihálka, Representations of Leibniz algebras, Algebr. Represent. Theory 18(2015) 477-490.

[41] I.N. Gel'fand, I.Ya. Dorfman, Hamiltonian operators and algebraic structures related to them, Funct. Anal. Appl. 13(1979) 248-262.

[42] J. Golenia, M. Pavlov, Z. Popowicz and A. Prykarpatsky, On a nonlocal Ostrovsky-Whitham type dynamical system, its Riemann type inhomogenious regularizations and their integrability. SIGMA 6(2010) 1-13.

[43] S. G'omez-Vidal, A.Kh. Khudoyberdiyev, B.A. Omirov, Some remarks on semisimple Leibniz algebras, J. Algebra 410(2014) 526-540. 
[44] D.D. Holm and R.I. Ivanov. Multi-component generalizations of the $\mathrm{CH}$ equation: geometrical aspects, peakons and numerical examples, J.Phys. A: Math. Theor. 43(2010) 492001 (20pp.).

[45] J. Hunter and R. Saxton, Dynamics of director fields SIAM J.Appl. Math. 51(1991) 1498-521.

[46] N. Jacobson, Lie algebras, Wiley (Interscience), New York, 1962.

[47] H. Kim, Complete left-invariant affine structures on nilpotent Lie groups, J. Diff. Geom. 24(1986) 373394.

[48] M. Ladra, I.M. Rikhsiboev, R.M. Turdibaev, Automorphisms and derivations of Leibniz algebras, (http://arxiv.org/abs1103.4721v1)[math.RA](24 Mar 2011).

[49] G. Leger, Derivations of Lie algebras. III, Duke Math. J. 30(1963) 637-645.

[50] J. Lewin, Subrings of finite index in finitely generated rings, J. Algebra 5(1967) 84-88.

[51] L.-C. Li, S. Parmentier, Nonlinear Poisson structures and $r$-matrices. Comm Math Phys 125(1989) $545-563$.

[52] A. Lichnerovich, Les varietes de Poisson at leurs algebres de la associees. J. Diff. Geometry, (2) 12(1977) 253-300.

[53] J.-L. Loday, Une version non commutative des algebres de Lie: les algebres de Leibniz, Eiseign. Math. (2) 39(1993) 269-293.

[54] G. Mason, G. Yamskulna, Leibniz algebras and Lie algebras, SIGMA Symmetry Integrability Geom. Methods Appl. 9(2013) Paper 063 (10pp.).

[55] W.A. Moens, A characterization of nilpotent Lie algebras by invertible Leibniz-derivations, Comm. Algebra 41(2013) 2427-2440.

[56] O.I. Mokhov, Symplectic and Poisson Geometry on Loop Spaces of Smooth Manifolds and Integrable Equations. Computer Science Institute Publisher, Moscow, 2004.

[57] D. Müller, Isometries of bi-invariant pseudo-Riemannian metrics of Lie groups, Geom. Dedicata 29(1989) 65-96.

[58] B.A. Omirov, On the derivations of filiform Leibniz algebras (Russian), Mat. Zametki 77(2005) 733742; translation in Math. Notes 77(2005) 677-685.

[59] W. Oevel, R-structures, Yang-Baxter equations and related involution theorems. J. Math. Phys. (5) 30(1989) 1140-1149.

[60] W. Oevel, Dirac constraints in field theory: Lifts of Hamiltonian systems to the cotangent bundle. J. Math. Phys. (1) 29(1988) 210-219.

[61] P. Olver, Applications of Lie Groups to Differential Equations. Second Edition, Springer-Verlag, New York, 1993.

[62] J.M. Osborn, Novikov algebras, Nova J. Algebra Geom. 1(1992) 1-14.

[63] M. Pavlov, The Gurevich-Zybin system J. Phys. A: Math. Gen. 38(2005) 3823-3840.

[64] A.M. Perea, Flat left-invariant connections adapted to the automorphism structure of a Lie group, J. Diff. Geom. 16(1981) 445-474.

[65] Z. Popowicz, A. Prykarpatsky, The non-polynomial conservation laws and integrability analysis of generalized Riemann type hydrodynamical equations. Nonlinearity 23(2010) 2517-2537.

[66] A.K. Prykarpatsky, O.D. Artemovych, Z. Popowicz and M.V. Pavlov, Differential-algebraic integrability analysis of the generalized Riemann type and Korteweg-de Vries hydrodynamical equations. J. Phys. A: Math. Theor. 43(2010) 295205 (13pp.).

[67] Y.A. Prykarpatsky, O.D. Artemovych, M. Pavlov and A.K. Prykarpatsky, The differential-algebraic and bi-Hamiltonian integrability analysis of the Riemann type hierarchy revisited. J. Math. Phys. 53(2012) 103521; arXiv:submit/0322023 [nlin.SI] 20 Sep 2011.

[68] Y.A. Prykarpatsky, O.D. Artemovych, M. Pavlov and A.K. Prykarpatsky, The differential-algebraic and bi-Hamiltoinian integrability analyss of the Riemann type hydrodynamic systems. Rep. Math. Phys. (3) 71(2013) 305-350.

[69] A. Prykarpatsky and I. Mykytyuk. Algebraic Integrability of Nonlinear Dynamical Systems on Manifolds: Classical and Quantum Aspects. Kluwer Academic Publishers, the Netherlands, 1998.

[70] A. Prykarpatsky, M. Prytula, The gradient-holonomic integrability analysis of a Whitham-type nonlinear dynamical model for a relaxing medium with spatial memory. Nonlinearity 19(2006) 2115-2122. 
[71] I.S. Rakhimov, K.K. Matsutova, B.A. Omirov, On derivations of semisimple Leibniz algebras, Bull. Malaysian Math. Sci. Soc. doi 10.1007/S40840-015-0113-5.

[72] I.S. Rakhimov, A.H. Al Nashri, K.A. Atan, Derivations of low-dimensional complex Leibniz algebras, JP J. Algebra Number Theory Appl. 21(2011) 69-81.

[73] I.S. Rakhimov, A.H. Al Nashri, Derivations of some classes of Leibniz algebras, J. Gen. Lie Theory Appl. 6(2012) Art. ID120501 (12pp.).

[74] C.B. Ray, A. Combs, N. Gin, A. Hedges, J.T. Hird and L. Zack, Nilpotent Lie and Leibniz algebras, Comm. Algebra 42(2014) 2404-2410.

[75] A.G. Reyman, M.A. Semenov-Tian-Shansky, Integrable Systems, The Computer Research Institute Publ., Moscow-Izhvek, 2003 (in Russian).

[76] M.A. Semenov-Tian-Shansky, What is a classical R-matrix? Func Anal Appl. (4) 17(1983) $259-272$.

[77] A. Sergyeyev, A simple way of making a Hamiltonian systems into a bi-Hamiltonian one. Acta Applicandae Math. (1) 83(2004) 183-197.

[78] I.A.B. Strachan, B.M. Szablikowski, Novikov Algebras and a Classification of Multicomponent Camassa-Holm Equations. Studies in Appl. Mathematics 133(2014) 84-117.

[79] S. Tôgô, On the derivation algebras of Lie algebras, Canad. J. Math. 13(1961) 201-216.

[80] O.O. Vaneeva, R.O. Popovych, C. Sophocleous, Equivalence transformation in the study of integrability. Phys. Scr. 89(2014) 038003 (9 pp.). 